\title{
One Water Molecule Stabilizes the Cationized Arginine Zwitterion
}

Matthew F. Bush, James S. Prell, Richard J. Saykally, and Evan R. Williams*

\section{Supporting Information}



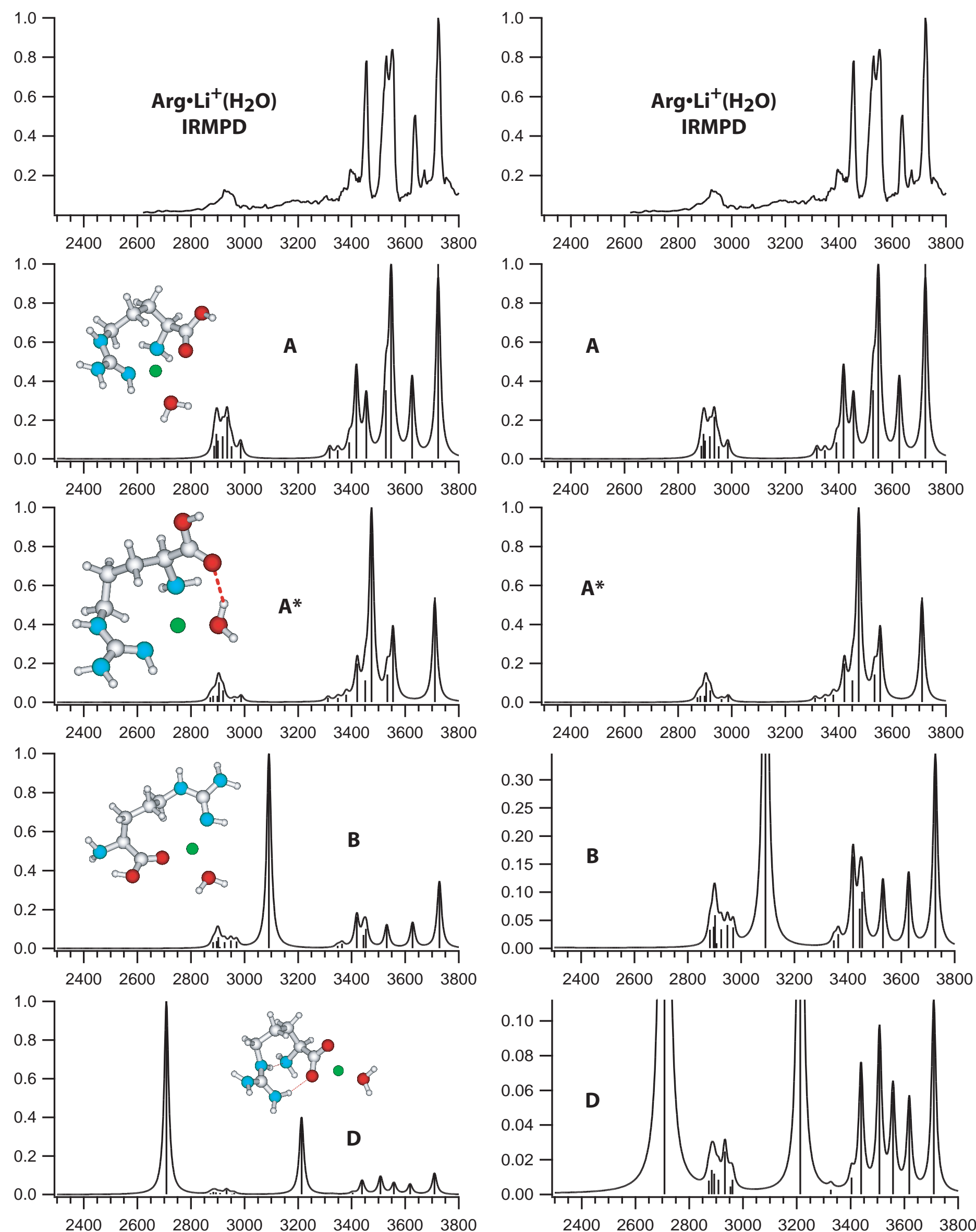

Photon energy $\left(\mathrm{cm}^{-1}\right)$

Supporting Information Figure 1. Photodissociation spectrum of $\mathrm{Arg}^{\circ} \mathrm{Li}^{+}\left(\mathrm{H}_{2} \mathrm{O}\right)$ and both unscaled intensity (left column) and scaled intensity (right column) B3LYP/6-31++G** calculated spectra for selected conformers of this ion. 

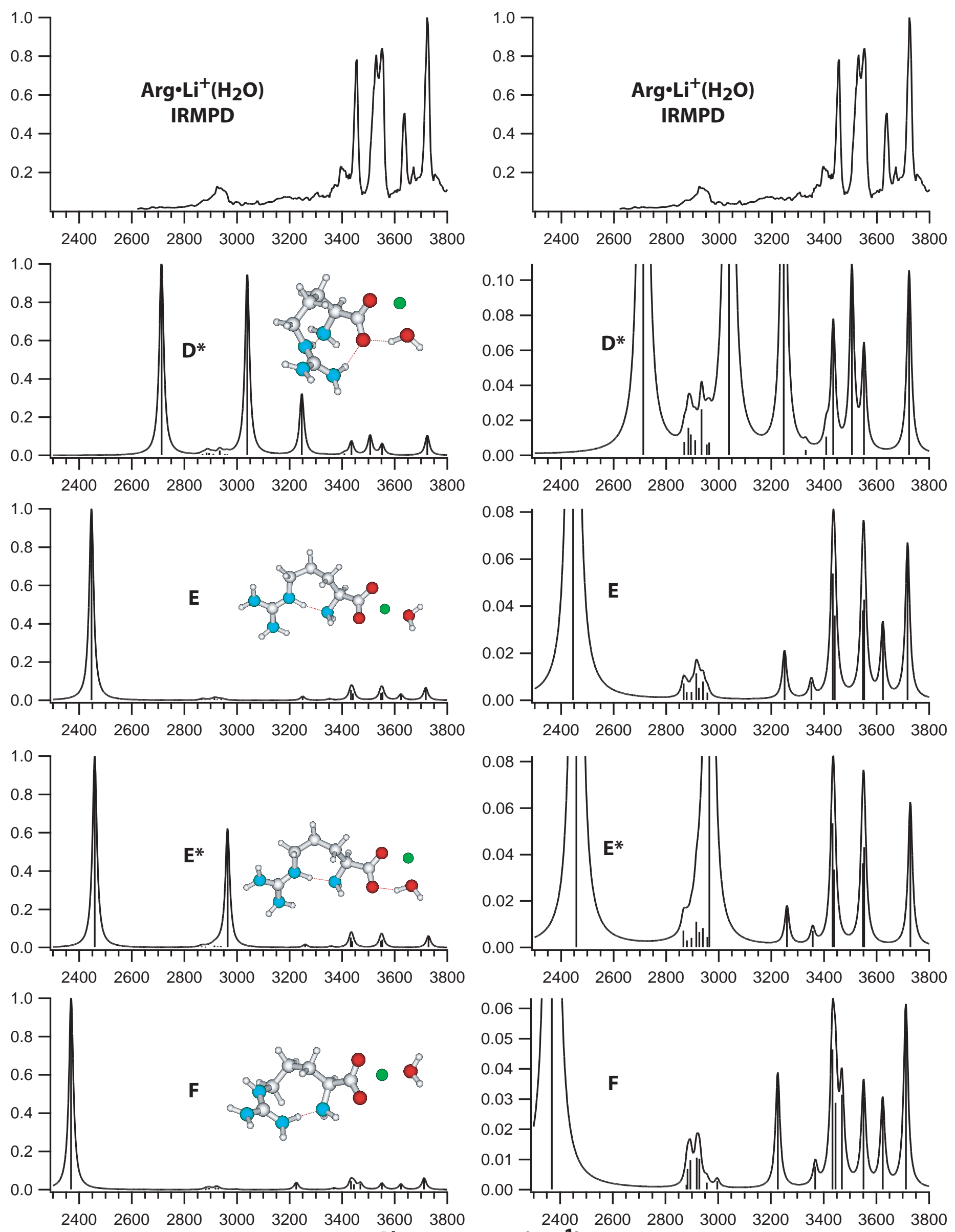

Photon energy $\left(\mathrm{cm}^{-1}\right)$

Supporting Information Figure 2. Photodissociation spectrum of $\mathrm{Arg} \cdot \mathrm{Li}^{+}\left(\mathrm{H}_{2} \mathrm{O}\right)$ and both unscaled intensity (left column) and scaled intensity (right column) B3LYP/6-31++G** calculated spectra for selected conformers of this ion. 

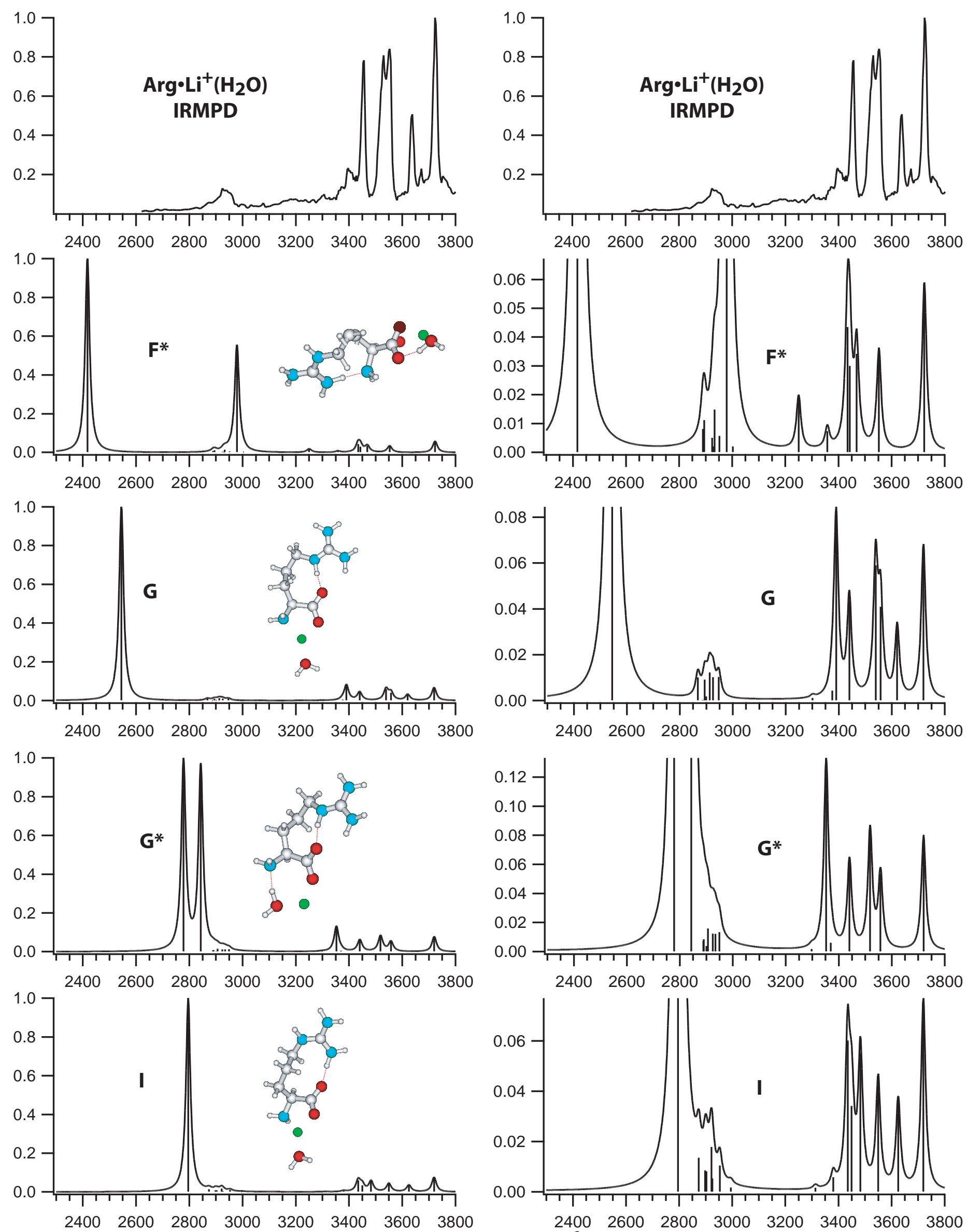

Photon energy $\left(\mathrm{cm}^{-1}\right)$

Supporting Information Figure 3. Photodissociation spectrum of $\operatorname{Arg}^{\circ} \mathrm{Li}^{+}\left(\mathrm{H}_{2} \mathrm{O}\right)$ and both unscaled intensity (left column) and scaled intensity (right column) B3LYP/6-31++G ${ }^{* *}$ calculated spectra for selected conformers of this ion. 

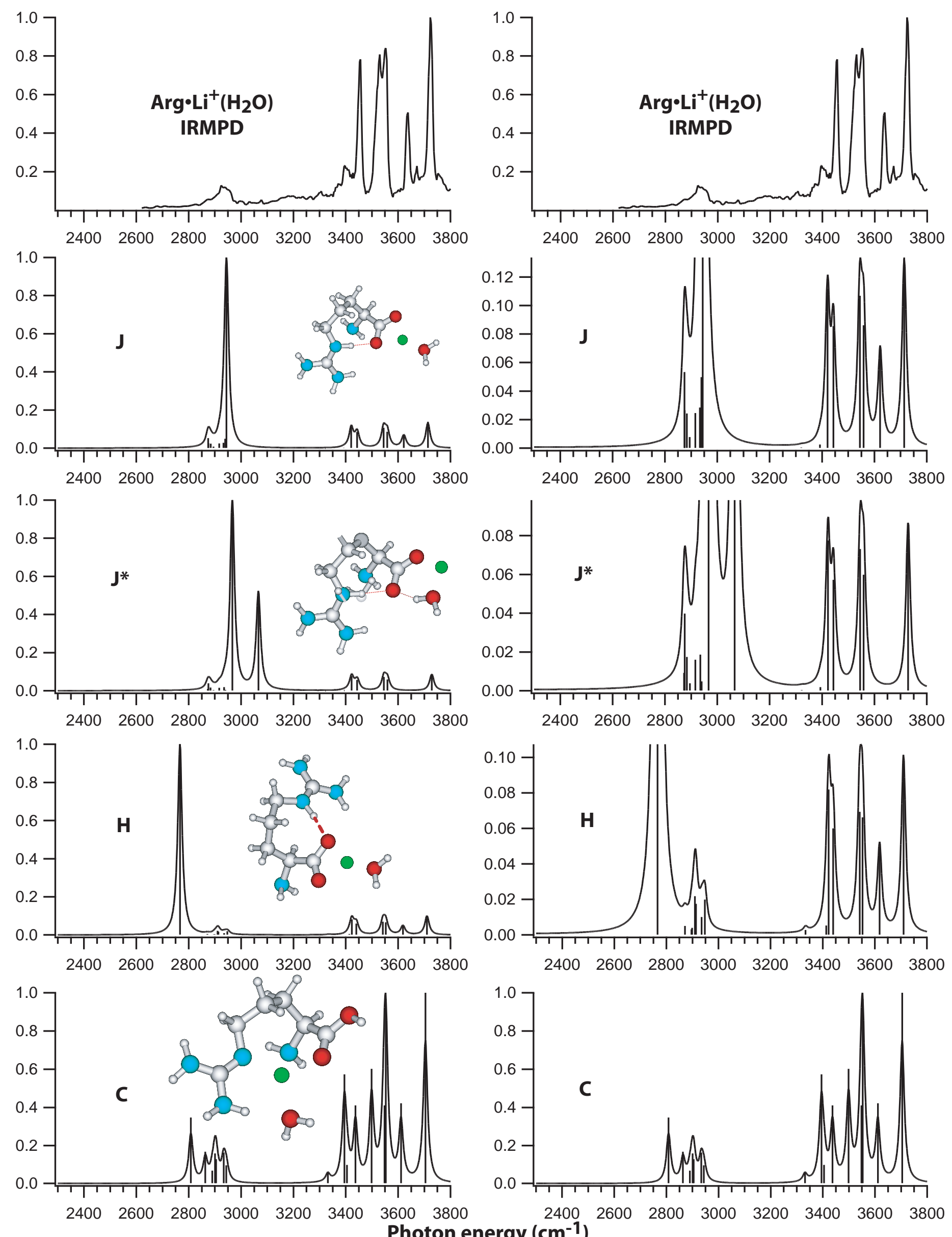

Supporting Information Figure 4. Photodissociation spectrum of $\mathrm{Arg}^{\circ} \mathrm{Li}^{+}\left(\mathrm{H}_{2} \mathrm{O}\right)$ and both unscaled intensity (left column) and scaled intensity (right column) B3LYP/6-31++ $G^{* *}$ calculated spectra for selected conformers of this ion. 


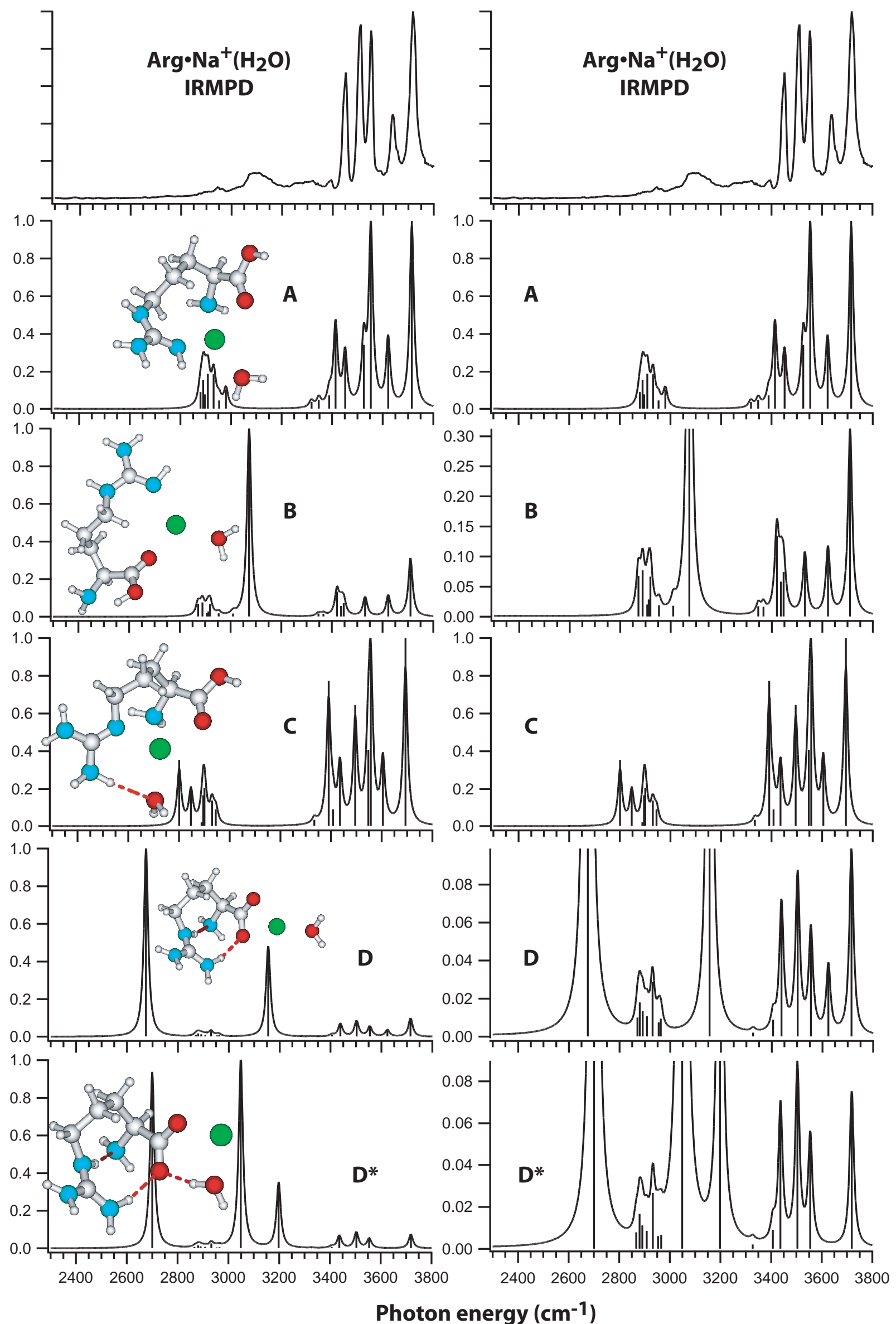

Supporting Information Figure 5. Photodissociation spectrum of $\mathrm{Arg}^{-\mathrm{Na}^{+}}\left(\mathrm{H}_{2} \mathrm{O}\right)$ and both unscaled intensity (left column) and scaled intensity (right column) B3LYP/6-31++G** calculated spectra for selected conformers of this ion. 


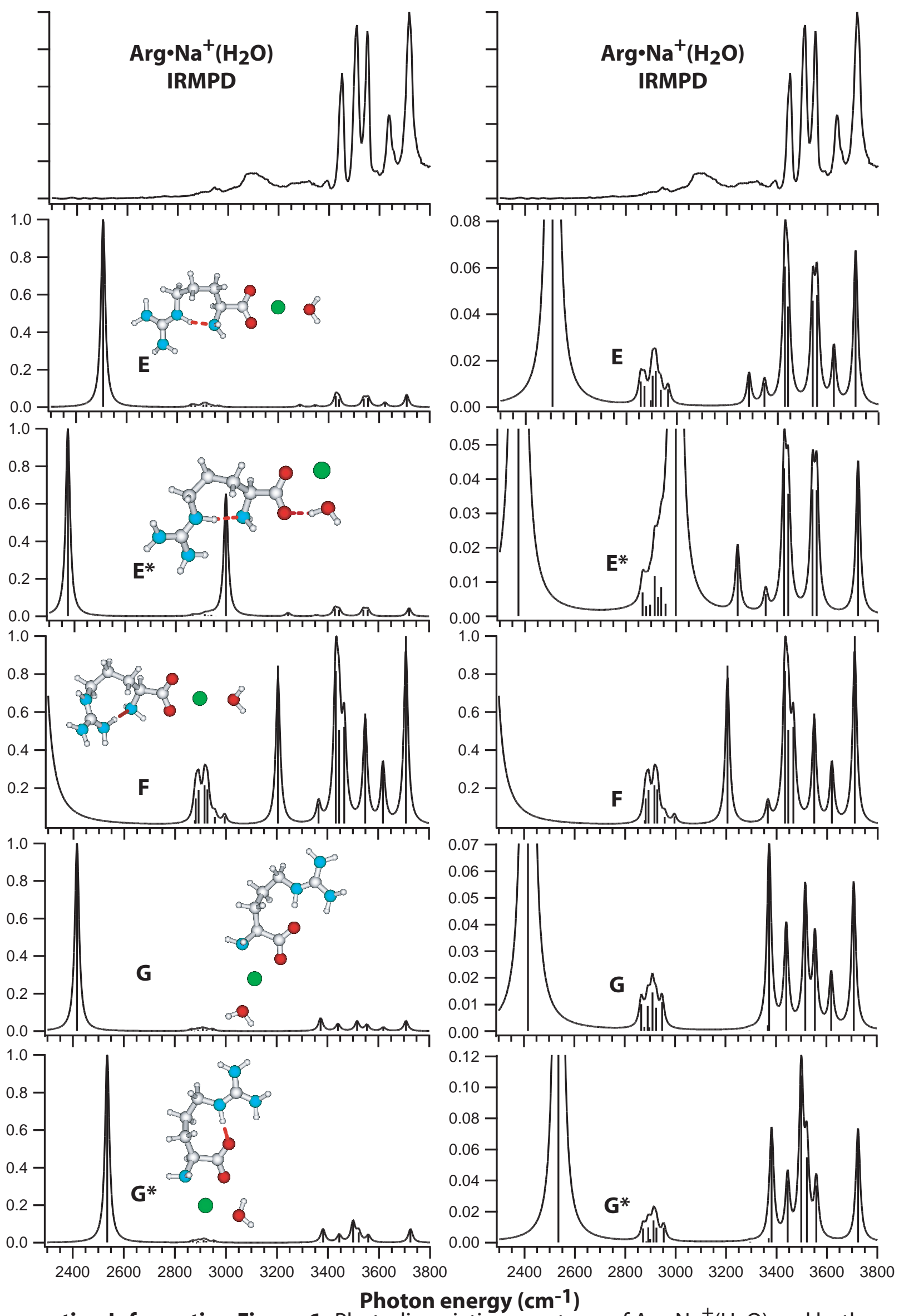

Supporting Information Figure 6. Photodissociation spectrum of $\mathrm{Arg}^{\circ} \mathrm{Na}^{+}\left(\mathrm{H}_{2} \mathrm{O}\right)$ and both unscaled intensity (left column) and scaled intensity (right column) B3LYP/6-31++G** calculated spectra for selected conformers of this ion. 


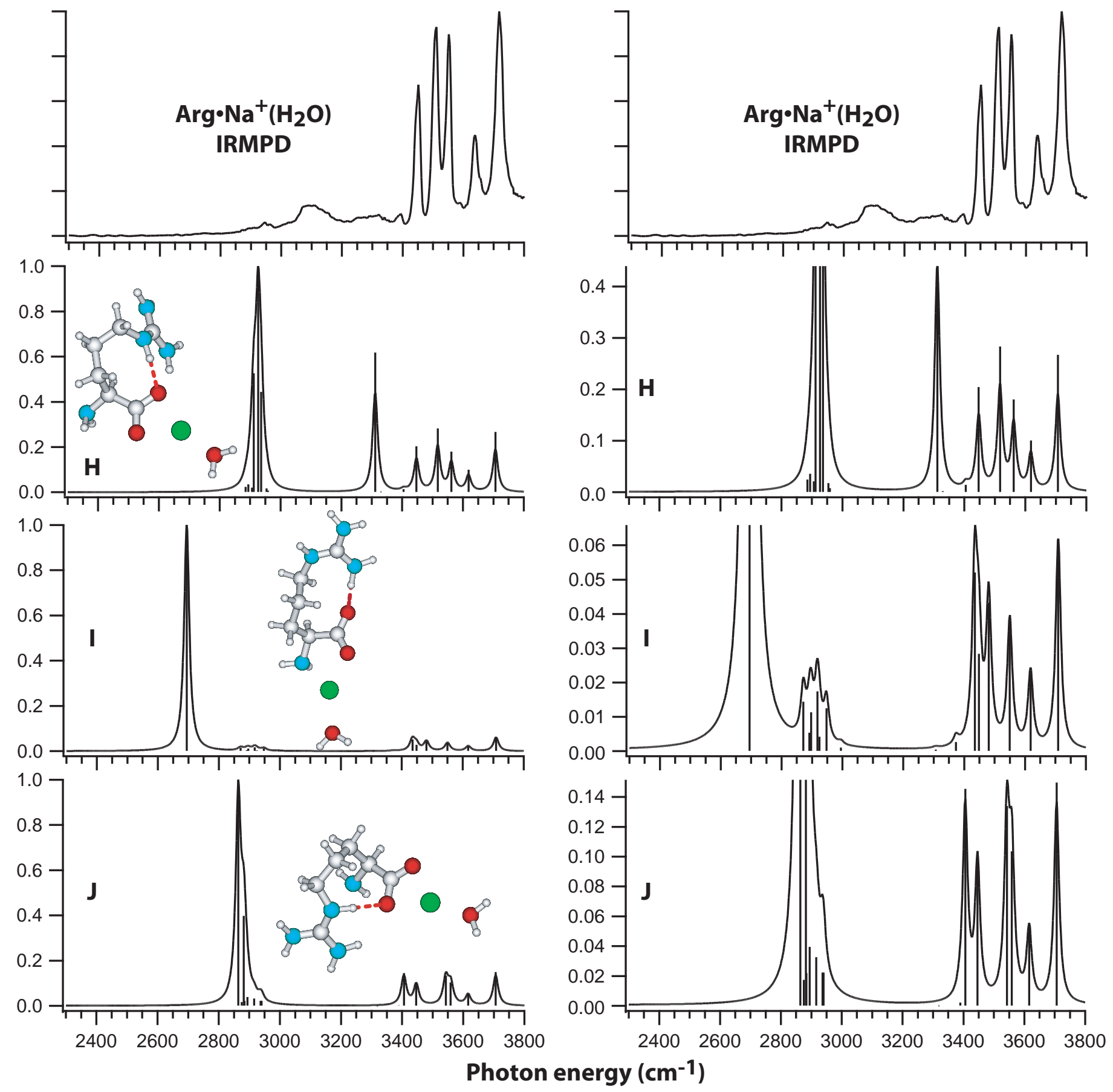

Supporting Information Figure 7. Photodissociation spectrum of $\operatorname{Arg}^{-N^{+}}\left(\mathrm{H}_{2} \mathrm{O}\right)$ and both unscaled intensity (left column) and scaled intensity (right column) B3LYP/6-31++ $\mathrm{G}^{* *}$ calculated spectra for selected conformers of this ion. 


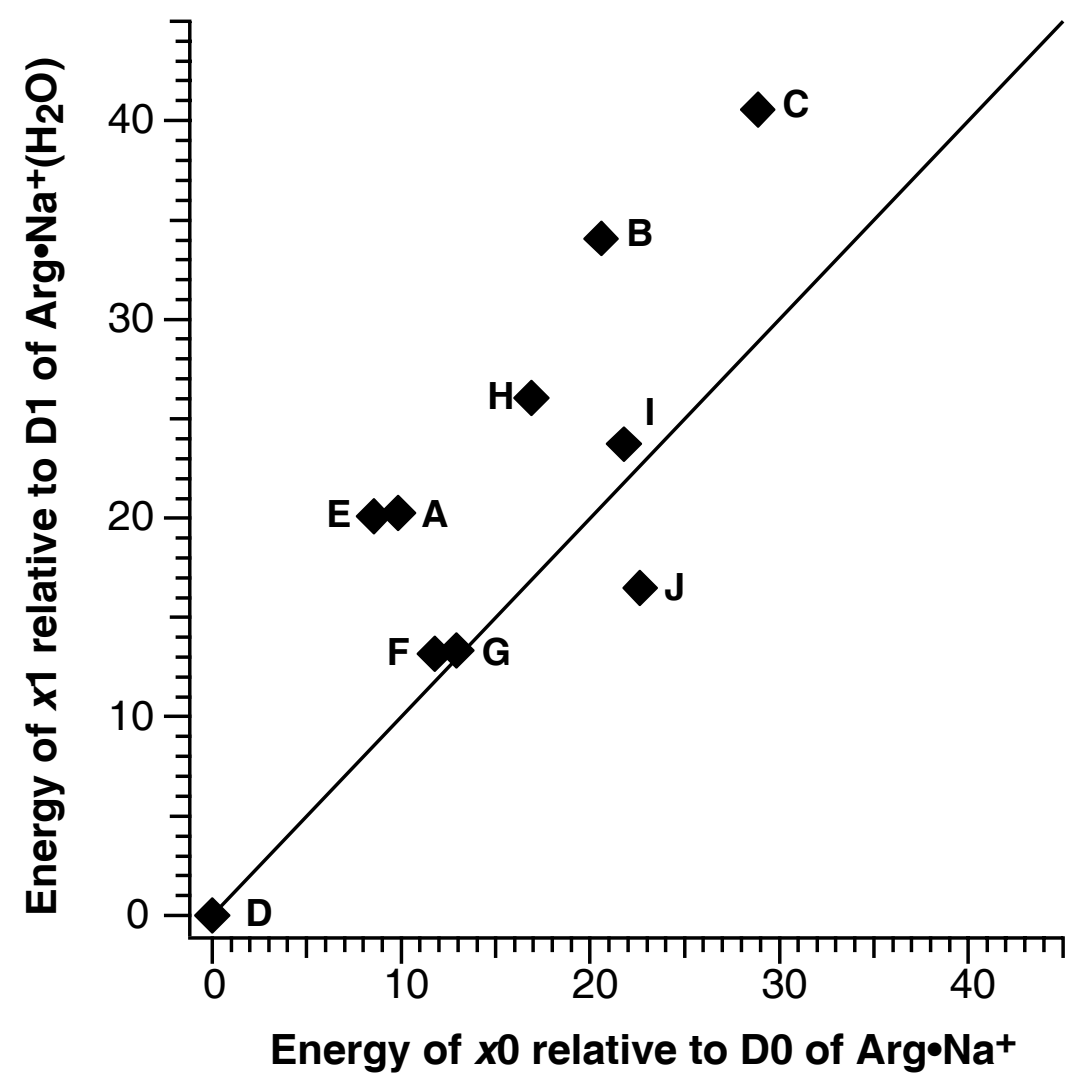

Supporting Information Figure 3. The B3LYP/6-31++ $\mathrm{G}^{* *}$ energies (in $\mathrm{kJ} / \mathrm{mol}$, including zero-point energies) of the $\boldsymbol{x} \mathbf{1}, \boldsymbol{x}=\mathbf{A}-\mathbf{J}$, structures of $\operatorname{Arg}^{\bullet} \mathrm{Na}^{+}\left(\mathrm{H}_{2} \mathrm{O}\right)$ plotted versus those of the corresponding $\mathbf{X} \mathbf{0}$ structures of $\mathrm{Arg} \bullet \mathrm{Na}^{+}$. The plotted line has a slope of unity. 


\section{Complete Citations}

(54) Shao, Y.; Molnar, L. F.; Jung, Y.; Kussmann, J.; Ochsenfeld, C.; Brown, S. T.; Gilbert, A. T. B.; Slipchenko, L. V.; Levchenko, S. V.; O'Neill, D. P.; DiStasio, R. A.; Lochan, R. C.; Wang, T.; Beran, G. J. O.; Besley, N. A.; Herbert, J. M.; Lin, C. Y.; Van Voorhis, T.; Chien, S. H.; Sodt, A.; Steele, R. P.; Rassolov, V. A.; Maslen, P. E.; Korambath, P. P.; Adamson, R. D.; Austin, B.; Baker, J.; Byrd, E. F. C.; Dachsel, H.; Doerksen, R. J.; Dreuw, A.; Dunietz, B. D.; Dutoi, A. D.; Furlani, T. R.; Gwaltney, S. R.; Heyden, A.; Hirata, S.; Hsu, C. P.; Kedziora, G.; Khalliulin, R. Z.; Klunzinger, P.; Lee, A. M.; Lee, M. S.; Liang, W.; Lotan, I.; Nair, N.; Peters, B.; Proynov, E. I.; Pieniazek, P. A.; Rhee, Y. M.; Ritchie, J.; Rosta, E.; Sherrill, C. D.; Simmonett, A. C.; Subotnik, J. E.; Woodcock, H. L.; Zhang, W.; Bell, A. T.; Chakraborty, A. K.; Chipman, D. M.; Keil, F. J.; Warshel, A.; Hehre, W. J.; Schaefer, H. F.; Kong, J.; Krylov, A. I.; Gill, P. M. W.; Head-Gordon, M. Advances in methods and algorithms in a modern quantum chemistry program package. Phys. Chem. Chem. Phys. 2006, 8, 3172-3191. 
Arginine $\bullet \mathrm{Li}^{+}\left(\mathrm{H}_{2} \mathrm{O}\right)\left(\mathrm{Arg} \cdot \mathrm{Li}^{+}\left(\mathrm{H}_{2} \mathrm{O}\right)\right)$

$\operatorname{Arg} \cdot \mathrm{Li}^{+}\left(\mathrm{H}_{2} \mathbf{O}\right)$ Structure A1

$\begin{array}{lrrr}\mathrm{C} & 0.8217645614 & 0.6571495300 & -1.7768408086 \\ \mathrm{C} & 1.0534013669 & 1.3178222105 & -3.1616736553 \\ \mathrm{C} & 0.8457291759 & 2.8436875669 & -3.2364865937 \\ \mathrm{C} & -0.6209002223 & 3.2895972331 & -3.4196169711 \\ \mathrm{~N} & -0.8264000268 & 4.7116510933 & -3.1904285774 \\ \mathrm{C} & -0.9968328538 & 5.2411810701 & -1.9408436673 \\ \mathrm{~N} & -1.1110845306 & 6.6140285870 & -1.9216772005 \\ \mathrm{~N} & -1.0206689858 & 4.4840345297 & -0.8747830671 \\ \mathrm{H} & -0.5328872432 & 5.3475062007 & -3.9184414036 \\ \mathrm{H} & -1.4969891388 & 7.0829750336 & -2.7293925518 \\ \mathrm{H} & -1.3197636436 & 7.0575909927 & -1.0397773219 \\ \mathrm{H} & -1.2621688075 & 5.0443116354 & -0.0591033263 \\ \mathrm{H} & 1.1263085563 & -0.3943670928 & -1.8635872343 \\ \mathrm{H} & 2.0787658814 & 1.0765644327 & -3.4574139729 \\ \mathrm{H} & 0.4044488326 & 0.8049528519 & -3.8843632643 \\ \mathrm{H} & 1.2530424836 & 3.3307718024 & -2.3428482785 \\ \mathrm{H} & 1.4308580513 & 3.2286720519 & -4.0795488893 \\ \mathrm{H} & -0.9575765143 & 3.0633313869 & -4.4370294968 \\ \mathrm{H} & -1.2857059891 & 2.7607026121 & -2.7355009210 \\ \mathrm{~N} & -0.5710258294 & 0.8067049351 & -1.3088158933 \\ \mathrm{H} & -1.2120129746 & 0.5642240694 & -2.0611473920 \\ \mathrm{H} & -0.7601833312 & 0.1419753298 & -0.5587925987 \\ \mathrm{C} & 1.7178089739 & 1.3023311258 & -0.7256153851 \\ \mathrm{O} & 2.9953161170 & 0.9497139430 & -0.8422234739 \\ \mathrm{O} & 1.3109208267 & 2.0818025406 & 0.1275358914 \\ \mathrm{H} & 3.5235037018 & 1.4121489156 & -0.1666918243 \\ \mathrm{Li} & -0.5972827213 & 2.6672006083 & -0.2010753969 \\ \mathrm{O} & -1.3732570763 & 2.3416972834 & 1.5852292263 \\ \mathrm{H} & -0.8019267851 & 2.2007888052 & 2.3520098524 \\ \mathrm{H} & -2.2698546076 & 2.4619801617 & 1.9228560600\end{array}$

Arg $\mathrm{Li}^{+}\left(\mathrm{H}_{2} \mathrm{O}\right)$ Structure $\mathrm{A1}$ *

$\begin{array}{lrll}\mathrm{C} & 0.9601508144 & 1.1901082967 & -1.7069158225 \\ \mathrm{C} & 1.3424574824 & 2.5283657402 & -2.3822718588 \\ \mathrm{C} & 0.6763687944 & 2.7746706136 & -3.7519489187 \\ \mathrm{C} & -0.6915428971 & 3.4848357009 & -3.7364759181 \\ \mathrm{~N} & -0.6336937823 & 4.8461537016 & -3.2137154644 \\ \mathrm{C} & -1.2704410085 & 5.2994030467 & -2.0945051442 \\ \mathrm{~N} & -1.3508153916 & 6.6685070722 & -2.0073113152 \\ \mathrm{~N} & -1.7091211582 & 4.4828941281 & -1.1677500394 \\ \mathrm{H} & -0.1876731416 & 5.5415231990 & -3.7953470425 \\ \mathrm{H} & -1.4078726499 & 7.2245412890 & -2.8486720221 \\ \mathrm{H} & -1.7833282127 & 7.0748827748 & -1.1915917384 \\ \mathrm{H} & -2.2668249838 & 5.0019339673 & -0.4920085383 \\ \mathrm{H} & 1.3084143631 & 0.3712573745 & -2.3531718081 \\ \mathrm{H} & 1.1163214875 & 3.3621789741 & -1.7051195846 \\ \mathrm{H} & 2.4283025480 & 2.5177188425 & -2.5116044869 \\ \mathrm{H} & 1.3581701485 & 3.3834754309 & -4.3575206721 \\ \mathrm{H} & 0.5687474319 & 1.8292877699 & -4.3015810182 \\ \mathrm{H} & -1.0734151233 & 3.5244944109 & -4.7638951339 \\ \mathrm{H} & -1.4346890329 & 2.9495969060 & -3.1443610947 \\ \mathrm{~N} & -0.4958682338 & 1.0921574352 & -1.4477895083 \\ \mathrm{H} & -0.6791769391 & 0.2735526400 & -0.8661843873\end{array}$




$\begin{array}{lr}\mathrm{H} & -\odot .9678687454 \\ \mathrm{C} & 1.7016949610 \\ \mathrm{O} & 3.0195440727 \\ \mathrm{O} & 1.1740983018 \\ \mathrm{H} & 3.4528794261 \\ \mathrm{Li} & -1.3448629679 \\ \mathrm{O} & -1.2532690779 \\ \mathrm{H} & -1.5954127374 \\ \mathrm{H} & -0.4252994577\end{array}$

0.9057216284

1. 0261084256

0.9144203347

0.9879733672

0.8087761401

2. 7127895955

2.1589965448

2. 3907950067

1.6538089452
$-2.3311203750$

$-\odot .3841991966$

$-0.5636925675$

0.7162940446

0.3022119383

$-0.4601323847$

1.3508561240

2. 2217410899

1.4625721999

\section{Arg $\cdot \mathrm{Li}^{+}\left(\mathrm{H}_{2} \mathrm{O}\right)$ Structure B1}

\begin{tabular}{|c|c|c|c|}
\hline C & 1.1021494580 & ๑.9590116689 & 0.6073529908 \\
\hline C & 2.0266576545 & 1.8482762666 & 1.4789137444 \\
\hline C & 2.7844090497 & 2.9913212578 & 0.7837965302 \\
\hline C & 1.8800145946 & 4. 0170421600 & 0.0788100828 \\
\hline $\mathrm{N}$ & 2.6053212160 & 5.1679885113 & $-\odot .4407717713$ \\
\hline C & 3.1123175645 & 5.2782496336 & $-1.7 \odot 26336157$ \\
\hline $\mathrm{N}$ & 3.6996974167 & 6.4960676246 & -1.9618246865 \\
\hline $\mathrm{N}$ & 3.0613005428 & 4.2953436441 & -2.5667570599 \\
\hline $\mathrm{H}$ & 2.9175754187 & 5.8541770006 & $0.231606 \odot 477$ \\
\hline $\mathrm{H}$ & 3. 3529898711 & 7.3201229074 & -1.4910390985 \\
\hline $\mathrm{H}$ & 4.0670050842 & 6.6594956527 & -2.8872094238 \\
\hline $\mathrm{H}$ & 3.3658842668 & 4.6321225644 & -3.4786284094 \\
\hline $\mathrm{H}$ & ๑.2855638006 & 1.5688333976 & 0.2034462902 \\
\hline $\mathrm{H}$ & 1.3924899465 & 2.2636232284 & 2.2727124576 \\
\hline $\mathrm{H}$ & 2.7581619347 & 1.1948320623 & 1.9713514742 \\
\hline $\mathrm{H}$ & 3. 3644398694 & 3.5097000363 & 1.5570600286 \\
\hline $\mathrm{H}$ & 3.5133659235 & 2.5949261882 & 0.0704808705 \\
\hline $\mathrm{H}$ & 1.3432385913 & 3.5641642117 & -0.7579932023 \\
\hline $\mathrm{H}$ & 1.1251855420 & 4. 3936889032 & 0.7798779463 \\
\hline $\mathrm{N}$ & $\odot .5936376492$ & -0.1762528755 & 1.3927464823 \\
\hline $\mathrm{H}$ & $\odot .7747995255$ & - ๑. ๑830๑०6122 & 2.3875762307 \\
\hline $\mathrm{H}$ & $-\odot .4 \odot \odot 72885 \odot 2$ & $-\odot .3375202012$ & 1.2635667044 \\
\hline C & 1.8511367662 & 0.3796670995 & -0.6046345634 \\
\hline 0 & 2.0793927884 & -0.9122483197 & -0.5530928978 \\
\hline 0 & 2.2179461234 & 1.0647127410 & -1.5559313143 \\
\hline $\mathrm{H}$ & 1.6266460606 & -1.2119811234 & 0.2873249907 \\
\hline $\mathrm{Li}$ & 2.7621119333 & 2.3858062381 & -2.7269409726 \\
\hline 0 & 3. 0708471286 & 1.3494238079 & -4.3187036618 \\
\hline $\mathrm{H}$ & 3. 3341469482 & 1.5011202938 & -5.2346459404 \\
\hline $\mathrm{H}$ & $2.980 \odot 291176$ & ๑. 3936821145 & -4.2011627942 \\
\hline
\end{tabular}

\section{$\operatorname{Arg} \cdot \mathrm{Li}^{+}\left(\mathrm{H}_{2} \mathrm{O}\right)$ Structure C1}

$\begin{array}{lrll}\mathrm{C} & 1.1863975332 & -1.2820382488 & -1.4093885075 \\ \mathrm{C} & 2.2966049456 & -1.6154226785 & -2.4410686058 \\ \mathrm{C} & 2.0927681772 & -1.2491094556 & -3.9275607899 \\ \mathrm{C} & 1.2073683979 & -2.1485259885 & -4.8104457648 \\ \mathrm{~N} & -0.2271093185 & -1.8495435542 & -4.7392809183 \\ \mathrm{C} & -0.9782533058 & -2.3547018493 & -5.6783744423 \\ \mathrm{H} & 0.3698716121 & -3.5918247807 & -6.6308346538 \\ \mathrm{H} & -1.2072081441 & -3.6507119741 & -7.2685429703 \\ \mathrm{H} & -2.8202675725 & -2.2010151731 & -6.5700222063 \\ \mathrm{H} & -2.6260850977 & -1.2862496157 & -5.1591481131 \\ \mathrm{H} & 1.6283022832 & -1.4043699052 & -0.4128095989 \\ \mathrm{H} & 3.1982528459 & -1.1018904351 & -2.0919069623 \\ \mathrm{H} & 2.5097378301 & -2.6888626302 & -2.3470886991 \\ \mathrm{H} & 1.7590644122 & -0.2095627370 & -4.0361661991\end{array}$




$\begin{array}{lrrr}\mathrm{H} & 3.0945388213 & -1.2801164336 & -4.3709487123 \\ \mathrm{H} & 1.5641934596 & -2.0044906003 & -5.8409670682 \\ \mathrm{H} & 1.4069302680 & -3.2044069417 & -4.5531317927 \\ \mathrm{Li} & -1.0190239674 & -1.0646692862 & -3.0875527253 \\ \mathrm{~N} & -0.0187565580 & -2.1139983636 & -1.5926461102 \\ \mathrm{H} & 0.2491810896 & -3.0654923526 & -1.8327301753 \\ \mathrm{H} & -0.5425682244 & -2.1740299575 & -0.7211236956 \\ \mathrm{C} & 0.7688939227 & 0.1765687603 & -1.5543681614 \\ \mathrm{O} & 1.6104112354 & 1.0230852630 & -0.9693452677 \\ \mathrm{O} & -0.2223416433 & 0.5374051253 & -2.1791639510 \\ \mathrm{H} & 1.3210626243 & 1.9387143146 & -1.1341655229 \\ \mathrm{~N} & -2.3370052438 & -2.0996262851 & -5.6876788225 \\ \mathrm{~N} & -0.5367060429 & -3.1576940236 & -6.6979898865 \\ \mathrm{O} & -2.9411088748 & -0.5513176821 & -3.0499870031 \\ \mathrm{H} & -3.7118566207 & -1.0807803117 & -2.8032222726 \\ \mathrm{H} & -3.1160304310 & 0.3508771432 & -2.7464861953\end{array}$

\section{Arg $\cdot \mathrm{Li}^{+}\left(\mathrm{H}_{2} \mathrm{O}\right)$ Structure D1}

$\begin{array}{lrrr}\mathrm{C} & -3.5563038229 & -2.5694707353 & 1.4190162887 \\ \mathrm{C} & -3.9034093596 & -1.7774336608 & 0.1317610558 \\ \mathrm{C} & -2.7922243743 & -0.9886487370 & -0.5950900918 \\ \mathrm{C} & -2.2345496550 & 0.2705509467 & 0.0941800476 \\ \mathrm{~N} & -1.5310485179 & -0.0731410384 & 1.3347308669 \\ \mathrm{C} & -0.2219311140 & -0.0747934019 & 1.5429325251 \\ \mathrm{~N} & 0.5999036669 & 0.7993056732 & 0.9201417449 \\ \mathrm{~N} & 0.2862734165 & -0.9736658151 & 2.4035887691 \\ \mathrm{H} & -2.1256885153 & -0.6396128926 & 1.9953729175 \\ \mathrm{H} & 0.2389308813 & 1.6555016999 & 0.5281251178 \\ \mathrm{H} & 1.6011877814 & 0.6835903788 & 0.9508685908 \\ \mathrm{H} & 1.2030053658 & -0.8573356439 & 2.8074341305 \\ \mathrm{H} & -0.2307610046 & -1.8491901973 & 2.5352476882 \\ \mathrm{H} & -4.3924919407 & -3.2638990750 & 1.5827643000 \\ \mathrm{H} & -4.2923833079 & -2.5095069386 & -0.5817957311 \\ \mathrm{H} & -4.7395145752 & -1.1048895315 & 0.3688030516 \\ \mathrm{H} & -1.9590056129 & -1.6600844747 & -0.8375120116 \\ \mathrm{H} & -3.2076270145 & -0.6611060485 & -1.5560501646 \\ \mathrm{H} & -1.5501927089 & 0.7811474562 & -0.5884059834 \\ \mathrm{H} & -3.0421954546 & 0.9709527516 & 0.3353768516 \\ \mathrm{C} & -2.3305311053 & -3.4736805254 & 1.2376895176 \\ \mathrm{O} & -2.3766555030 & -4.3601881234 & 0.3437762408 \\ \mathrm{O} & -1.3133142593 & -3.3517460179 & 2.0138831002 \\ \mathrm{~N} & -3.3714149308 & -1.6792798074 & 2.5829380494 \\ \mathrm{H} & -3.0612423483 & -2.2160242594 & 3.3903195533 \\ \mathrm{H} & -4.2533120040 & -1.2357824869 & 2.8321423476 \\ \mathrm{Li} & -0.7832808696 & -5.0339925133 & 1.1235202997 \\ \mathrm{O} & 0.2181800279 & -6.6394086207 & 0.9834118959 \\ \mathrm{H} & 0.8988600270 & -7.0121498110 & 1.5590015083 \\ \mathrm{H} & -0.0072485508 & -7.3241972201 & 0.3386544691\end{array}$

\section{Arg $\cdot \mathrm{Li}^{+}\left(\mathrm{H}_{2} \mathrm{O}\right)$ Structure $\mathrm{D1}^{*}$}

$\begin{array}{ll}\mathrm{C} & -3.8179100644 \\ \mathrm{C} & -4.1233073428 \\ \mathrm{C} & -5.1143767978 \\ \mathrm{C} & -4.6735981888 \\ \mathrm{~N} & -4.5140720773 \\ \mathrm{C} & -5.3291677249 \\ \mathrm{~N} & -6.0353248670\end{array}$
$-0.3257015235$
0.0219007317
1.1615415144
2. 6053578711
2. 8176370904
3.4826520155
4.5536509748 


$\begin{array}{ll}\mathrm{N} & -5.4390505542 \\ \mathrm{H} & -3.8569786732 \\ \mathrm{H} & -5.7579303857 \\ \mathrm{H} & -6.7781051400 \\ \mathrm{H} & -5.8267419683 \\ \mathrm{H} & -5.2540045582 \\ \mathrm{H} & -3.3393697012 \\ \mathrm{H} & -4.5259325202 \\ \mathrm{H} & -3.1648995150 \\ \mathrm{H} & -6.0739196296 \\ \mathrm{H} & -5.3151977573 \\ \mathrm{H} & -5.4193512200 \\ \mathrm{H} & -3.7206568841 \\ \mathrm{~N} & -2.9351923120 \\ \mathrm{H} & -2.8239647102 \\ \mathrm{H} & -2.0100612982 \\ \mathrm{C} & -5.0902547399 \\ \mathrm{O} & -5.8946689476 \\ \mathrm{O} & -5.2476104202 \\ \mathrm{Li} & -7.2543605939 \\ \mathrm{O} & -7.2970843430 \\ \mathrm{H} & -6.5234145042 \\ \mathrm{H} & -7.4781223109\end{array}$

\section{$\operatorname{Arg} \cdot \mathrm{Li}^{+}\left(\mathrm{H}_{2} \mathrm{O}\right)$ Structure E1}

$\begin{array}{lrr}\mathrm{C} & -0.9220340200 & -1.8323674417 \\ \mathrm{C} & -1.6732384071 & -0.6210661272 \\ \mathrm{C} & -2.2619600585 & 0.3706849872 \\ \mathrm{C} & -1.3937947528 & 1.5904811569 \\ \mathrm{~N} & -0.1136237836 & 1.1847169484 \\ \mathrm{C} & 0.7319219510 & 2.0061467405 \\ \mathrm{~N} & 0.5310919835 & 3.3399872924 \\ \mathrm{~N} & 1.8348718253 & 1.5160403293 \\ \mathrm{H} & 0.1641853683 & 0.1625731824 \\ \mathrm{H} & -0.2546202580 & 3.7577893150 \\ \mathrm{H} & 1.0565954922 & 3.9265960317 \\ \mathrm{H} & 2.6293071083 & 2.1128153035 \\ \mathrm{H} & 2.0084413188 & 0.5222414148 \\ \mathrm{H} & -1.5932835507 & -2.3307962562 \\ \mathrm{H} & -1.0142276652 & -0.0930343234 \\ \mathrm{H} & -2.4910689317 & -1.0339562794 \\ \mathrm{H} & -3.2022843280 & 0.7722116077 \\ \mathrm{H} & -2.5204634419 & -0.1537482982 \\ \mathrm{H} & -1.2104765884 & 2.1710650621 \\ \mathrm{H} & -1.9346532107 & 2.2300906731 \\ \mathrm{C} & -0.6140751451 & -2.8396675168 \\ \mathrm{O} & -1.5755213449 & -3.4471631242 \\ \mathrm{O} & 0.5991886066 & -3.0041070635 \\ \mathrm{~N} & 0.3222855218 & -1.4418042075 \\ \mathrm{H} & 1.0821587390 & -1.5826985315 \\ \mathrm{H} & 0.5037287334 & -2.1250484114 \\ \mathrm{Li} & -0.1909299387 & -4.2617547662 \\ \mathrm{O} & 0.0963319754 & -5.5735594739 \\ \mathrm{H} & 0.9362837211 & -5.8606247816 \\ \mathrm{H} & -0.5958810913 & -6.1152562834\end{array}$

$-2.6534895705$

$-1.0108188177$

$-0.1257397000$

$-1.5133017643$

$-3.3672343400$

$-2.8547988949$

$-0.6115263694$

1.3069340792

1.3553303453

0.6794360797

2. 2541218961

1.2677578109

1.3635349010

$-1.2546598128$

$-2.2459094746$

$-0.8307115999$

$-1.4688201896$

$-1.0556121710$

$-2.5255916178$

$-2.0417596841$

$-3.6215091663$

$-3.4362810619$

$-4.5661670703$
1.0560034212

0.4618258028

1.4921267892

1. 8268319045

2. 4196215204

3. 0102522543

3. 0466083746

3. 6267744911

2. 2595654731

2. 5735408171

3. 6767513890

3. 8031531278

3.6092407838

1. 7636110100

$-0.2419452532$

$-0.1353598929$

1. 0993509995

2. 4206286610

0.9111910187

2. 5355679134

$-0.0704084083$

$-0.6215008919$

$-0.4208730669$

1.7715341652

1. 0967250074

2. 5061331537

$-1.6705675060$

$-3.0156148700$

$-3.3967348492$

$-3.4177879786$

\section{$\operatorname{Arg} \cdot \mathrm{Li}^{+}\left(\mathrm{H}_{2} \mathrm{O}\right)$ Structure $\mathrm{E} 1^{*}$}




\begin{tabular}{|c|c|}
\hline $\mathrm{C}$ & -1.0254568010 \\
\hline C & -1.6804576087 \\
\hline C & -2.2031276983 \\
\hline C & -1.2476553974 \\
\hline $\mathrm{N}$ & $-\odot .0190649987$ \\
\hline C & $\odot .8674549875$ \\
\hline $\mathrm{N}$ & $\odot .7692594038$ \\
\hline $\mathrm{N}$ & 1.9071832131 \\
\hline $\mathrm{H}$ & $\odot .1887146605$ \\
\hline $\mathrm{H}$ & $\odot .0437621428$ \\
\hline $\mathrm{H}$ & 1.3178663313 \\
\hline $\mathrm{H}$ & 2.7415621052 \\
\hline $\mathrm{H}$ & 1.9984235445 \\
\hline $\mathrm{H}$ & -1.7308676762 \\
\hline $\mathrm{H}$ & $-\odot .9785006432$ \\
\hline $\mathrm{H}$ & -2.5236955527 \\
\hline $\mathrm{H}$ & -3.1020499690 \\
\hline $\mathrm{H}$ & -2.5175714561 \\
\hline $\mathrm{H}$ & $-\odot .9970463401$ \\
\hline $\mathrm{H}$ & -1.7522437133 \\
\hline C & $-\odot .7973903607$ \\
\hline 0 & -1.8391149059 \\
\hline 0 & $\odot .3908371303$ \\
\hline $\mathrm{N}$ & ๑.2426876929 \\
\hline $\mathrm{H}$ & ๑. 9888301968 \\
\hline H & 0.3937705613 \\
\hline Li & -1.6758912749 \\
\hline 0 & $\odot .0822857825$ \\
\hline $\mathrm{H}$ & 0.7845394918 \\
\hline $\mathrm{H}$ & $\odot .4594693076$ \\
\hline
\end{tabular}

$-2.0052499416$

$-0.7338133333$

๑. 2877951410

1.4307377590

$\odot .9191037950$

1. 6625969149

3. 0068333166

1. 0775968181

$-\odot .1154256751$

3. 4948117730

3. 5407618225

1. 6055068246

0.0729583056

$-2.4558759335$

$-0.2488755038$

$-1.0750628756$

0.7682591109

$-\odot .2273855110$

2. 0025931016

2. 1054782180

$-3.0261801989$

$-3.5622121834$

$-3.2307735258$

$-1.7118359091$

$-1.8628025239$

$-2.4347400633$

$-4.7924069472$

$-5.0122528394$

$-5.4573479948$

$-4.3578089607$
0.8364818650

0.2533179683

1.2902200918

1.6568810896

2. 2756178330

2. 9085380205

2. 9643607624

3. 5510105364

2. 0951967390

2. 4632544002

3.6215658657

3.7597395479

3. 5291039898

1.5426366881

$-0.4397283533$

$-0.3535841222$

$\odot .8890844927$

2.2065817744

$\odot .7517324590$

2.3595173938

$-0.3026969061$

$-0.7969155058$

$-0.6695066642$

1.5539809432

0.8672684257

2. 2561841376

$-2.1158118718$

$-2.5617099496$

$-3.0451015847$

$-1.8994715087$

\section{Arg $\cdot \mathrm{Li}^{+}\left(\mathrm{H}_{2} \mathrm{O}\right)$ Structure $\mathrm{F} 1$}

$\begin{array}{llll}\mathrm{C} & -1.3497315328 & -2.2396569229 & 1.4449814669 \\ \mathrm{C} & -2.1026508314 & -0.9011839392 & 1.3217158350 \\ \mathrm{C} & -3.6319287486 & -1.0018012095 & 1.1970533771 \\ \mathrm{C} & -4.4189700356 & -1.3857409919 & 2.4601983125 \\ \mathrm{~N} & -4.3123229342 & -0.3948344782 & 3.5460112604 \\ \mathrm{C} & -3.5858043550 & -0.5129685737 & 4.6676861146 \\ \mathrm{~N} & -2.6921040824 & -1.4843817083 & 4.8121155822 \\ \mathrm{~N} & -3.7874249584 & 0.3899681028 & 5.6570656211 \\ \mathrm{H} & -4.8227844314 & 0.4697323997 & 3.4239837982 \\ \mathrm{H} & -2.2795225358 & -2.0389168007 & 3.9874879953 \\ \mathrm{H} & -2.2755449773 & -1.6221139227 & 5.7225132450 \\ \mathrm{H} & -3.1605044075 & 0.4419442299 & 6.4452361604 \\ \mathrm{H} & -4.6267182172 & 0.9483241656 & 5.6959610264 \\ \mathrm{H} & -1.6879082566 & -2.8935687859 & 0.6300991383 \\ \mathrm{H} & -1.8270271225 & -0.2502284185 & 2.1626916165 \\ \mathrm{H} & -1.7210712552 & -0.4120181265 & 0.4209738878 \\ \mathrm{H} & -4.0173462347 & -0.0385454429 & 0.8383936054 \\ \mathrm{H} & -3.8917232191 & -1.7329584272 & 0.4200412111 \\ \mathrm{H} & -5.4827925307 & -1.4689959043 & 2.2155842228 \\ \mathrm{H} & -4.1091324987 & -2.3509054172 & 2.8623789737 \\ \mathrm{C} & 0.1601951455 & -2.0253020615 & 1.2045845604 \\ \mathrm{O} & 0.5323825011 & -1.4368964401 & 0.1510229394 \\ \mathrm{O} & 0.9808996080 & -2.4653304590 & 2.0741937871 \\ \mathrm{~N} & -1.5494819230 & -2.9198559417 & 2.7545499364 \\ \mathrm{H} & -0.6031502692 & -3.1333893051 & 3.0921872556\end{array}$




$\begin{array}{lrrr}\text { H } & -2.0076416993 & -3.8190436034 & 2.6202252123 \\ \text { Li } & 2.2969477745 & -1.8204893488 & 0.8054008735 \\ \mathrm{O} & 4.1645519692 & -1.7591354021 & 0.4538624107 \\ \mathrm{H} & 4.5885462874 & -1.3996948022 & -0.3367251211 \\ \mathrm{H} & 4.8650114434 & -2.1385421989 & 1.0010573573\end{array}$

\section{Arg $\mathrm{Li}^{+}\left(\mathrm{H}_{2} \mathrm{O}\right)$ Structure $\mathrm{F}^{*}$ *}

$\begin{array}{lr}\mathrm{C} & -0.4686363417 \\ \mathrm{C} & -1.5419986935 \\ \mathrm{C} & -1.5267829374 \\ \mathrm{C} & -0.3840835010 \\ \mathrm{~N} & -0.4048010862 \\ \mathrm{C} & 0.4214078690 \\ \mathrm{~N} & 1.2230578866 \\ \mathrm{~N} & 0.4201136441 \\ \mathrm{H} & -1.1218433035 \\ \mathrm{H} & 1.0838616099 \\ \mathrm{H} & 1.9451769225 \\ \mathrm{H} & 0.8810484849 \\ \mathrm{H} & 0.0424487741 \\ \mathrm{H} & -0.7290775115 \\ \mathrm{H} & -2.5132711400 \\ \mathrm{H} & -1.4801063340 \\ \mathrm{H} & -1.5006833013 \\ \mathrm{H} & -2.4769366046 \\ \mathrm{H} & 0.5969277449 \\ \mathrm{H} & -0.4648505656 \\ \mathrm{C} & -0.5428914419 \\ \mathrm{O} & 0.4339479894 \\ \mathrm{O} & -1.6055750540 \\ \mathrm{~N} & 0.9092578512 \\ \mathrm{H} & 1.4061162817 \\ \mathrm{H} & 1.3894899373 \\ \mathrm{Li} & -1.7530182267 \\ \mathrm{O} & -0.2823874521 \\ \mathrm{H} & 0.2126098166 \\ \mathrm{H} & 0.2827779817 \\ & \\ & \end{array}$

\section{Arg $\cdot \mathrm{Li}^{+}\left(\mathrm{H}_{2} \mathrm{O}\right)$ Structure $\mathrm{G1}$}

C $\quad-3.9360274098$

C $\quad-4.4550395360$

C $\quad-4.1034248200$

$\mathrm{N} \quad-4.7340376867$

C $\quad-5.0135267112$

$\mathrm{N} \quad-4.5561065367$

$\mathrm{N} \quad-5.8005504671$

H $\quad-5.1264722248$

H $\quad-3.8941493178$

H $\quad-4.8749725305$

H $\quad-5.8484529960$

H $\quad-6.1497789619$

H $\quad-5.7919005750$

$\mathrm{H} \quad-3.2336135846$

H $\quad-3.3577066396$

H $\quad-5.5450138840$

H $\quad-4.0421901358$
$-2.3126076836$

$-1.2006393083$

$-0.1534625763$

$\odot .8737175600$

1.7732874271

1.7369425874

0.6996183219

2.7906644827

2.4866602255

$-0.2614692785$

$\odot .7879750485$

2.7346339370

3.6859110123

$-3.0373617516$

$-1.7036803904$

$-0.7081768635$

$-0.6531933162$

0.3954844173

0.4002011956

1. 5121257597

$-3.0598907927$

$-2.9400272796$

$-3.7209732511$

$-1.8111379112$

$-1.8834332127$

$-2.4660312986$

$-4.7286243959$

$-4.4877549976$

$-3.8634613200$

$-4.7787229223$
1.4992402010

1.5580270549

0.4303138657

0.4377676248

1.6064327464

2. 6619419673

2. 8774838339

3. 5124775352

1. 6166564233

2. 4216779546

3. 5788295794

4. 4077230298

3.2409476007

2. 2769560126

1.5288644739

2. 5369617643

$-0.5455701123$

0.4579488906

0.3970379959

$-0.4478647956$

0.1496202046

$-0.6395572988$

$-0.0716439683$

1.7550241025

0.8606337697

2. 3700855104

$-1.5693768019$

$-2.6314369845$

$-2.0210747049$

$-3.3537144832$
$-0.2687701644$

0.5091425972

1. 3774349419

2. 8710329148

3. 4230535436

4.6834021109

5.6999608281

4.9568967179

2. 7078012192

5.5341572299

6.6477910386

5.8831788998

4.1828908039

$-0.5981358167$

$-0.2294122457$

1.1207248751

1. 2906724153

$\odot .9991104303$
$-0.8028283336$

$-0.0190567693$

1.1665985364

1. 0805880867

$-0.1163694288$

$-\odot .3780342831$

๑. 3861769152

$-1.4398808411$

$-0.7979232156$

1. 1269554481

0.2482359061

$-1.8326873921$

$-1.9939792338$

$-0.0918255054$

$\odot .3828250526$

$-0.7230820698$

1. 2532786356

2. 1074649463 


$\begin{array}{ll}\mathrm{H} & -4.4715707001 \\ \mathrm{H} & -3.0126652509 \\ \mathrm{~N} & -4.5087785734 \\ \mathrm{H} & -4.2469653546 \\ \mathrm{H} & -3.6477467245 \\ \mathrm{C} & -5.7954584497 \\ \mathrm{O} & -6.3760709274 \\ \mathrm{O} & -5.8203869392 \\ \mathrm{Li} & -5.9591932271 \\ \mathrm{O} & -6.7316537961 \\ \mathrm{H} & -6.5916069432 \\ \mathrm{H} & -7.4241841813\end{array}$

\section{Arg $\cdot \mathrm{Li}^{+}\left(\mathrm{H}_{2} \mathrm{O}\right)$ Structure $\mathrm{G1}^{\text {* }}$}

$\begin{array}{lrr}\mathrm{C} & -5.1132236445 & -0.4615042599 \\ \mathrm{C} & -4.0671680195 & 0.1974160755 \\ \mathrm{C} & -4.6624714783 & 1.0698160953 \\ \mathrm{C} & -4.1393210298 & 2.5160731280 \\ \mathrm{~N} & -4.5008969630 & 3.1807014876 \\ \mathrm{C} & -4.9395936581 & 4.4162109869 \\ \mathrm{~N} & -4.7273096423 & 5.3882196213 \\ \mathrm{~N} & -5.6384097714 & 4.7006798427 \\ \mathrm{H} & -4.7163543825 & 2.5346247887 \\ \mathrm{H} & -4.0674786030 & 5.2610615767 \\ \mathrm{H} & -5.2063136302 & 6.2737289805 \\ \mathrm{H} & -5.6960845977 & 5.6436776921 \\ \mathrm{H} & -5.8712961747 & 3.9349444643 \\ \mathrm{H} & -5.8578498513 & -0.9778061879 \\ \mathrm{H} & -3.4680970014 & -0.6049606857 \\ \mathrm{H} & -3.3733874483 & 0.7804032799 \\ \mathrm{H} & -5.7564785878 & 1.1049472391 \\ \mathrm{H} & -4.4392570895 & 0.6141821761 \\ \mathrm{H} & -4.5865328619 & 3.0499766004 \\ \mathrm{H} & -3.0493324168 & 2.5247549031 \\ \mathrm{~N} & -4.5213444742 & -1.5005726623 \\ \mathrm{H} & -3.9955966786 & -2.1670929800 \\ \mathrm{H} & -3.8434507759 & -1.0712389671 \\ \mathrm{C} & -5.9084740595 & 0.5288266832 \\ \mathrm{O} & -5.4556474071 & 1.6897722558 \\ \mathrm{O} & -6.9633484318 & 0.0776116608 \\ \mathrm{Li} & -7.6517882982 & -1.3147305609 \\ \mathrm{O} & -6.5113725175 & -2.7425675692 \\ \mathrm{H} & -6.3475965623 & -3.6237214575 \\ \mathrm{H} & -5.6957987911 & -2.4016347887\end{array}$

\section{Arg $\cdot \mathrm{Li}^{+}\left(\mathrm{H}_{2} \mathbf{0}\right)$ Structure $\mathrm{H1}$}

$\begin{array}{ll}\mathrm{C} & -1.7836234353 \\ \mathrm{C} & -2.7212272537 \\ \mathrm{C} & -2.8995530655 \\ \mathrm{C} & -3.5054450570 \\ \mathrm{~N} & -2.5153240990 \\ \mathrm{C} & -2.7601104784 \\ \mathrm{~N} & -3.9430338445 \\ \mathrm{~N} & -1.8070691795 \\ \mathrm{H} & -1.5552253826 \\ \mathrm{H} & -4.6680478412 \\ \mathrm{H} & -4.1845482140\end{array}$

3. 3847252488

3. 0031970301

$-2.1890997989$

$-1.3034367856$

0.5496024760

$-0.1254145416$

1.8043758157

$-1.8816106431$

$-3.2043922812$

$-4.1543480836$

$-2.9543045733$
$-1.5137455349$
$-0.3947605024$

$-1.5811261883$

$-2.7236128313$

$-2.3418243027$

$-1.6369989253$

$-1.0640060607$

$-1.2081429591$

$-0.2965063563$

$-1.5182383364$

$-1.7906764266$

$-0.6362792643$
1.9781356510

1.0351534513

$-1.4448962024$

$-0.7288332629$

$-1.9520720953$

$-1.8938529922$

$-2.7862008401$

$-1.8004886293$

$-2.8651127914$

$-3.9983728737$

$-4.0994229134$

$-4.6261928313$
$-1.1106112749$

$-0.1896400052$

0.9540060802

$\odot .9921884064$

$-0.2627595979$

$-0.4337039707$

0.4773851687

$-1.5521789646$

$-1.0600317343$

1. 2285963465

0.4164335050

$-1.9053585463$

$-2.1795341209$

$-0.4930495498$

๑. 2566020376

- $\odot .8080409296$

$\odot .8782421437$

1.9238394683

1.8383025796

1.1212941329

$-1.9991144677$

$-1.4339947781$

$-2.6299070167$

$-2.0234316125$

$-2.1994084394$

$-2.5510024211$

$-3.3756867549$

$-3.4022816880$

$-3.7535220099$

$-2.9129039440$
๑. 8491140927

0.4976289137

1. 5144590858

2. 8733494863

3. 7013230474

4.8651233064

5.4947560723

5.4409328292

3.2925449632

5. 1060425869

6. 2898569563 


$\begin{array}{lr}\mathrm{H} & -1.8458146397 \\ \mathrm{H} & -0.9204005312 \\ \mathrm{H} & -2.1880532277 \\ \mathrm{H} & -3.6954342780 \\ \mathrm{H} & -2.3573058049 \\ \mathrm{H} & -3.5769329596 \\ \mathrm{H} & -1.9556088518 \\ \mathrm{H} & -4.3860394705 \\ \mathrm{H} & -3.8262397301 \\ \mathrm{C} & -0.3790701866 \\ \mathrm{O} & -0.1495081291 \\ \mathrm{O} & 0.5280545676 \\ \mathrm{~N} & -1.8034336060 \\ \mathrm{H} & -1.2920442664 \\ \mathrm{H} & -1.3476646239 \\ \mathrm{Li} & 1.6404861209 \\ \mathrm{O} & 3.4355726131 \\ \mathrm{H} & 3.9135704923 \\ \mathrm{H} & 4.0539069809\end{array}$

$-0.0690903766$

$-0.1824671748$

0.1309916367

$-1.1358922353$

$-2.0218778108$

$-3.4577537167$

$-3.2549712394$

$-1.7011763683$

$-3.2550314133$

$-0.8951026002$

$-1.2548556519$

$-0.9723721170$

0.5380287997

0.1499351813

1. 4132360368

$-1.6534692385$

$-2.2401607211$

$-2.6283385910$

$-2.2111966972$
6.4227969294

4.9694163479

1.7218765802

0.2672662545

$-0.4386820826$

1.0651533183

1.6868302931

2. 7266758153

3. 3916596031

1. 2295907471

2. 4583131699

$\odot .3658073378$

$-\odot .2688787675$

$-1.0587905811$

$-0.0258084386$

1. 7490582379

1.9135864372

2.6580321764

1. 1701368908

\section{Arg $\bullet \mathrm{Li}^{+}\left(\mathrm{H}_{2} \mathbf{0}\right)$ Structure I1}

$\begin{array}{lrrr}\mathrm{C} & -1.3077682657 & -1.3340048906 & -0.5015197869 \\ \mathrm{C} & -2.1079368632 & -1.4325440594 & 0.8189088841 \\ \mathrm{C} & -1.5098970998 & -0.6952731506 & 2.0302468791 \\ \mathrm{C} & -1.3554925928 & 0.8217111767 & 1.8278781537 \\ \mathrm{~N} & -0.5989821304 & 1.4689231376 & 2.9114133375 \\ \mathrm{C} & 0.7315888229 & 1.6614478779 & 2.9258810666 \\ \mathrm{~N} & 1.5176533681 & 1.1787661342 & 1.9748725667 \\ \mathrm{~N} & 1.2578885384 & 2.3695576093 & 3.9554946344 \\ \mathrm{H} & -1.0906023614 & 1.6556974095 & 3.7748585448 \\ \mathrm{H} & 1.2172860320 & 0.4596667733 & 1.2722801379 \\ \mathrm{H} & 2.4855850986 & 1.4658556371 & 1.9416479883 \\ \mathrm{H} & 2.2574875071 & 2.4588025942 & 4.0565587578 \\ \mathrm{H} & 0.6859108506 & 3.0035616178 & 4.4930746408 \\ \mathrm{H} & -1.3944204421 & -0.3178782652 & -0.9031134722 \\ \mathrm{H} & -3.1291398301 & -1.0719720633 & 0.6314284376 \\ \mathrm{H} & -2.1954924882 & -2.4933464124 & 1.0868497449 \\ \mathrm{H} & -2.1557938742 & -0.8784509162 & 2.8976609155 \\ \mathrm{H} & -0.5322019702 & -1.1238167912 & 2.2699373514 \\ \mathrm{H} & -0.8373853211 & 1.0402861754 & 0.8934796405 \\ \mathrm{H} & -2.3325958927 & 1.3133674497 & 1.7868459091 \\ \mathrm{C} & 0.2037369684 & -1.6048889267 & -\odot .2526439985 \\ \mathrm{O} & 0.8782970551 & -0.6285632003 & 0.1564023281 \\ \mathrm{O} & 0.6221344598 & -2.7812608128 & -0.4499346739 \\ \mathrm{~N} & -1.8574284060 & -2.3244094713 & -1.4728023155 \\ \mathrm{H} & -1.7311021570 & -1.9722979152 & -2.4217256655 \\ \mathrm{H} & -2.8646300998 & -2.4199270851 & -1.3504196728 \\ \mathrm{Li} & -0.5207227109 & -3.8967047778 & -1.2902478428 \\ \mathrm{O} & -0.1911753034 & -5.6683393592 & -1.9212688693 \\ \mathrm{H} & 0.6835677675 & -6.0660079838 & -1.8126568215 \\ \mathrm{H} & -0.7236616954 & -6.2961373178 & -2.4253038217\end{array}$

\section{$\operatorname{Arg} \cdot \mathrm{Li}^{+}\left(\mathrm{H}_{2} \mathrm{O}\right)$ Structure J1}

$\begin{array}{llr}\text { C } & -2.0504298309 & -1.8710411688 \\ \mathrm{C} & -2.8304431516 & -0.6464568266 \\ \mathrm{C} & -2.0736412076 & 0.4775992928 \\ \mathrm{C} & -1.0493177352 & 1.3075948981\end{array}$

1.6221682639

1.0770178192

$\odot .3376832552$

1.1267693910 


$\begin{array}{lrrr}\mathrm{N} & 0.2072304058 & 0.5696956794 & 1.3057479214 \\ \mathrm{C} & 1.1645895077 & 0.9040455373 & 2.1497018018 \\ \mathrm{~N} & 1.2548488655 & 2.1468660806 & 2.6663023428 \\ \mathrm{~N} & 2.0743930913 & -0.0223774991 & 2.5184390895 \\ \mathrm{H} & 0.2109528620 & -0.4218003434 & 0.9925240980 \\ \mathrm{H} & 0.6727506762 & 2.8951579346 & 2.3241908673 \\ \mathrm{H} & 1.8903524119 & 2.3552962680 & 3.4207648988 \\ \mathrm{H} & 2.9781357970 & 0.2406883771 & 2.8795656331 \\ \mathrm{H} & 1.9352790778 & -0.9800591614 & 2.2260285720 \\ \mathrm{H} & -2.7984995015 & -2.6527517334 & 1.8108020242 \\ \mathrm{H} & -3.5867601103 & -1.0301060947 & 0.3842632491 \\ \mathrm{H} & -3.3837472515 & -0.2133905491 & 1.9215927430 \\ \mathrm{H} & -1.5886537247 & 0.0849617989 & -0.5651302261 \\ \mathrm{H} & -2.8314905834 & 1.1829210210 & -0.0209979429 \\ \mathrm{H} & -0.8472365456 & 2.2305047270 & 0.5678498305 \\ \mathrm{H} & -1.4553251880 & 1.5829048929 & 2.1089536765 \\ \mathrm{C} & -1.1266481017 & -2.4534367025 & 0.5464531377 \\ \mathrm{O} & 0.1014303343 & -2.0573577587 & 0.4640866565 \\ \mathrm{O} & -1.5904879108 & -3.2929513598 & -0.2656051486 \\ \mathrm{~N} & -1.2909440986 & -1.5103640064 & 2.8302907220 \\ \mathrm{H} & -0.8252708513 & -2.3315455190 & 3.2114036726 \\ \mathrm{H} & -1.9380435058 & -1.1958110689 & 3.5506449019 \\ \mathrm{Li} & 0.1480069936 & -3.3325613866 & -1.0344361082 \\ \mathrm{O} & 1.0795496857 & -4.2273975796 & -2.4239488119 \\ \mathrm{H} & 2.0003004311 & -4.1592297871 & -2.7080931908 \\ \mathrm{H} & 0.6649033094 & -4.9009675388 & -2.9805184791\end{array}$

\section{Arg $\mathrm{Li}^{+}\left(\mathrm{H}_{2} \mathrm{O}\right)$ Structure $\mathrm{J1}^{\text {* }}$}

$\begin{array}{lrrr}\mathrm{C} & -2.0952807555 & -2.1629314695 & 1.3178479668 \\ \mathrm{C} & -2.8240609531 & -0.8876123577 & 0.8191958854 \\ \mathrm{C} & -2.0192781029 & 0.2363546760 & 0.1318393934 \\ \mathrm{C} & -0.9773011705 & 0.9987177704 & 0.9645552932 \\ \mathrm{~N} & 0.2471178148 & 0.2066774057 & 1.1348499209 \\ \mathrm{C} & 1.2120275442 & 0.4805312801 & 1.9918977435 \\ \mathrm{~N} & 1.3513131699 & 1.7061366092 & 2.5375758133 \\ \mathrm{~N} & 2.0807671952 & -0.4910112665 & 2.3428707628 \\ \mathrm{H} & 0.2130242665 & -0.7733255911 & 0.7946690244 \\ \mathrm{H} & 0.7962347739 & 2.4837145473 & 2.2162374928 \\ \mathrm{H} & 1.9809942635 & 1.8664868109 & 3.3085580618 \\ \mathrm{H} & 2.9913682458 & -0.2739741410 & 2.7172585506 \\ \mathrm{H} & 1.9020357138 & -1.4365814048 & 2.0335975697 \\ \mathrm{H} & -2.8753692758 & -2.9209265984 & 1.4707166882 \\ \mathrm{H} & -3.5892198867 & -1.2127192908 & 0.1063953434 \\ \mathrm{H} & -3.3680546984 & -0.4685555957 & 1.6768717667 \\ \mathrm{H} & -1.5357839066 & -0.1382311605 & -0.7792926143 \\ \mathrm{H} & -2.7473519523 & 0.9815973225 & -0.2073804969 \\ \mathrm{H} & -0.7316332016 & 1.9317234268 & 0.4411923840 \\ \mathrm{H} & -1.3878522253 & 1.2552485833 & 1.9500977883 \\ \mathrm{C} & -1.1830750700 & -2.7383727911 & 0.2214234425 \\ \mathrm{O} & -1.7253505372 & -3.5163556646 & -0.6140862665 \\ \mathrm{O} & 0.0448280974 & -2.3890846220 & 0.1861957812 \\ \mathrm{~N} & -1.3342117670 & -1.8818626378 & 2.5453822315 \\ \mathrm{H} & -0.9043277740 & -2.7355333896 & 2.8955796399 \\ \mathrm{H} & -1.9747581461 & -1.5709521650 & 3.2731044236 \\ \mathrm{Li} & -0.8673084674 & -4.3563180876 & -1.9617744929 \\ \mathrm{O} & 0.8809367672 & -3.8026853941 & -1.9083955344 \\ \mathrm{H} & 1.7851531837 & -3.8624673128 & -2.2317679889\end{array}$


Arginine $\bullet \mathrm{Na}^{+}\left(\mathrm{H}_{2} \mathrm{O}\right)\left(\mathrm{Arg} \cdot \mathrm{Na}^{+}\left(\mathrm{H}_{2} \mathrm{O}\right)\right)$

\section{Arg $\cdot \mathrm{Na}^{+}\left(\mathrm{H}_{2} \mathrm{O}\right)$ Structure $\mathrm{A1}$}

$\begin{array}{lrr}\mathrm{C} & -1.8682455379 & 0.5793021116 \\ \mathrm{C} & -3.0943231151 & -0.3437334109 \\ \mathrm{C} & -4.4823435139 & 0.3284133058 \\ \mathrm{C} & -5.1249674526 & 0.5416657328 \\ \mathrm{~N} & -6.4110148503 & 1.2198476014 \\ \mathrm{C} & -6.5456207663 & 2.5827885331 \\ \mathrm{~N} & -7.8534432147 & 3.0090945581 \\ \mathrm{~N} & -5.5096325015 & 3.3751882847 \\ \mathrm{H} & -7.1989320864 & 0.6814109990 \\ \mathrm{H} & -8.5872334620 & 2.4329530481 \\ \mathrm{H} & -8.0270443399 & 4.0011800938 \\ \mathrm{H} & -5.8252627749 & 4.3414080888 \\ \mathrm{H} & -0.9746534456 & -0.0508473992 \\ \mathrm{H} & -2.9238680456 & -0.8644362520 \\ \mathrm{H} & -3.0666532467 & -1.1200490276 \\ \mathrm{H} & -4.4418628909 & 1.2869444471 \\ \mathrm{H} & -5.1531259670 & -0.3093221135 \\ \mathrm{H} & -5.2821572918 & -0.4287157846 \\ \mathrm{H} & -4.4761602537 & 1.1244935677 \\ \mathrm{C} & -1.7884007667 & 1.6344890232 \\ \mathrm{O} & -1.4035714340 & 1.1152633545 \\ \mathrm{O} & -2.0551366244 & 2.8188332159 \\ \mathrm{H} & -1.3888403705 & 1.8178639115 \\ \mathrm{~N} & -1.9134447341 & 1.2662283502 \\ \mathrm{H} & -0.9906695064 & 1.6388515055 \\ \mathrm{H} & -2.1149661263 & 0.5842560122 \\ \mathrm{Na} & -3.1822886669 & 3.4112814642 \\ \mathrm{O} & -2.0880992582 & 5.2230728928 \\ \mathrm{H} & -2.2223966982 & 5.6494887404 \\ \mathrm{H} & -1.4425885910 & 5.7698959854\end{array}$

\section{Arg $\bullet \mathrm{Na}^{+}\left(\mathrm{H}_{2} \mathrm{O}\right)$ Structure B1}

$\begin{array}{ll}\mathrm{C} & -2.1191476039 \\ \mathrm{C} & -2.3645175869 \\ \mathrm{C} & -3.4609835058 \\ \mathrm{C} & -4.8568937597 \\ \mathrm{~N} & -4.8495776514 \\ \mathrm{C} & -5.7436128370 \\ \mathrm{~N} & -5.9022691501 \\ \mathrm{~N} & -6.3241360807 \\ \mathrm{H} & -4.4162548870 \\ \mathrm{H} & -5.8167391438 \\ \mathrm{H} & -6.5170081531 \\ \mathrm{H} & -7.0328190968 \\ \mathrm{H} & -1.3735480411 \\ \mathrm{H} & -2.6016168188 \\ \mathrm{H} & -1.4050928558 \\ \mathrm{H} & -3.1442032199 \\ \mathrm{H} & -3.5714933869 \\ \mathrm{H} & -5.5742527105 \\ \mathrm{H} & -5.2010284486\end{array}$

๑. 0522876735

$-1.1200738065$

$-0.9011947068$

$-1.4358981098$

$-2.8943113484$

$-3.6252341923$

$-4.9222066819$

$-3.1341789077$

$-3.3867051964$

$-5.1373819210$

$-5.5305231024$

$-3.7877281531$

$-0.2826458028$

$-2.0138076302$

$-1.3174632912$

$-1.3800076033$

0.1619330637

$-1.1143829911$

$-1.0304638372$
5.2045184962

4.9799454806

4.9483789801

6.3336309947

6.2826823494

6.2502385057

6.1306642552

6. 3036517030

5.9507530793

6.5202632935

6.1963680157

6. 3738065790

5. 0959314084

4. 0330574685

5.7567099394

4.4172420351

4.3605531639

6.8186157011

6.9896084772

4.1048700043

2.9373249724

4. 2507602848

2. 2630307676

6.5094694611

6.7324261532

7.2379866052

6.2021755694

7.1196442652

7.9762054682

6.6521127633
6.2125507659

7.1888036114

8.2583850085

7. 8952688701

7.7859888414

7.0441059314

7. 4818698909

5.9811861782

8.5556714458

8. 4651007990

6.9623562070

5.6520205624

5. 4814929246

6.6032374763

7.6789721719

9.1936603188

8.5092239607

8.6633999588

6.9454174190 


$\begin{array}{ll}\mathrm{C} & -3.3696837602 \\ \mathrm{O} & -3.7037261907 \\ \mathrm{O} & -4.0054255377 \\ \mathrm{H} & -3.0110039538 \\ \mathrm{~N} & -1.6487326220 \\ \mathrm{H} & -0.6888793012 \\ \mathrm{H} & -1.7223390768 \\ \mathrm{Na} & -5.7754154379 \\ \mathrm{O} & -6.7911844944 \\ \mathrm{H} & -7.5869701814 \\ \mathrm{H} & -6.4666191833\end{array}$

๑. 3909072765

1.6654713046

$-0.4556085936$

2. 1017581344

1. 3005165271

1.5238937969

1. 2877223439

$-1.6539879328$

$-0.9466586754$

$-1.2368439332$

$-\odot .1767590761$
5.3780759355

5.3742556286

4.7595928410

5.9505653072

6.8410826914

6.5994500101

7.8541208586

4.2801968714

2. 3674646308

1.9027756665

1.8804617523

\section{Arg $\cdot \mathrm{Na}^{+}\left(\mathrm{H}_{2} \mathrm{O}\right)$ Structure C1}

$\begin{array}{lrrr}\mathrm{C} & 2.1254092435 & -1.1297097678 & -1.8380388197 \\ \mathrm{C} & 1.9501528485 & 0.4103216939 & -1.9403337278 \\ \mathrm{C} & 0.7590267802 & 1.1005040596 & -1.2297127951 \\ \mathrm{C} & -0.5440025304 & 1.3100053756 & -2.0221164433 \\ \mathrm{~N} & -1.4355863474 & 0.1485875439 & -2.0587919280 \\ \mathrm{C} & -2.5326341918 & 0.2782519828 & -2.7478101423 \\ \mathrm{H} & -2.2814685571 & 2.1793407708 & -3.5102988856 \\ \mathrm{H} & -3.6338446570 & 1.3485628604 & -4.1271857019 \\ \mathrm{H} & -4.3830326526 & -0.5533357359 & -3.0385036425 \\ \mathrm{H} & -3.2891395095 & -1.5411910925 & -2.2137600794 \\ \mathrm{H} & 3.1249972815 & -1.3516721120 & -2.2326025968 \\ \mathrm{H} & 2.8758831428 & 0.8386671433 & -1.5433306676 \\ \mathrm{H} & 1.9424226061 & 0.6613993713 & -3.0094787326 \\ \mathrm{H} & 0.5290538188 & 0.6162003110 & -0.2718157576 \\ \mathrm{H} & 1.1100486181 & 2.1033545269 & -0.9621811226 \\ \mathrm{H} & -1.0648857555 & 2.1592641865 & -1.5521488648 \\ \mathrm{H} & -0.2675184911 & 1.6370448548 & -3.0410684215 \\ \mathrm{Na} & -0.7946597077 & -1.8637036077 & -0.9979250626 \\ \mathrm{~N} & 1.0740950476 & -1.8781415989 & -2.5468934387 \\ \mathrm{H} & 1.4302984298 & -2.7831592455 & -2.8473952072 \\ \mathrm{H} & 0.7979283431 & -1.3789297356 & -3.3887997372 \\ \mathrm{C} & 2.1272429669 & -1.5467728957 & -0.3681869591 \\ \mathrm{O} & 3.3112702627 & -1.3470818690 & 0.2127050191 \\ \mathrm{O} & 1.1551161411 & -1.9791619338 & 0.2370079459 \\ \mathrm{H} & 3.2513629310 & -1.5862665229 & 1.1549985401 \\ \mathrm{~N} & -3.4121706275 & -0.7839374955 & -2.8741539880 \\ \mathrm{~N} & -2.9292823301 & 1.4149914574 & -3.4075300376 \\ \mathrm{O} & -2.6712877877 & -3.2762536155 & -0.9143308329 \\ \mathrm{H} & -3.2124575914 & -3.4110511574 & -\odot .1231453670 \\ \mathrm{H} & -2.7421301871 & -4.0998068172 & -1.4181131088\end{array}$

\begin{tabular}{|c|c|c|c|}
\hline \multicolumn{4}{|c|}{ Arg $\cdot \mathrm{Na}^{+}\left(\mathrm{H}_{2} \mathrm{O}\right)$ structure D1 } \\
\hline $\mathrm{c}$ & -0.6658026555 & 0.4193851812 & $-\odot .2960817657$ \\
\hline C & $\odot .3823447527$ & -0.3282964230 & 0.5494707963 \\
\hline 0 & 0.1257684160 & $-\odot .4808700837$ & 1.7695926513 \\
\hline C & -2.0669346488 & -0.2 & $-\odot$ \\
\hline C & -2.2007046473 & -1.7582503504 & $-\odot .4927258555$ \\
\hline C & -1.9574480204 & -2.2675157344 & -1.9256335751 \\
\hline $\mathrm{N}$ & $-\odot .5648055596$ & -2.0487076054 & -2.3320918220 \\
\hline C & ๑. 3948468883 & -2.9573321411 & -2.4310421215 \\
\hline $\mathrm{N}$ & 0.1338253847 & -4.2310290233 & -2.8050255960 \\
\hline $\mathrm{N}$ & 1.65603 & -2.5 & -2.1516655453 \\
\hline $\mathrm{H}$ & $-\odot .2657410381$ & $-1.0<$ & -2.2283116717 \\
\hline $\mathrm{H}$ & $-\odot .7102190748$ & -4.4555328013 & -3.3089626869 \\
\hline
\end{tabular}




$\begin{array}{lrrr}\mathrm{H} & 0.8345564477 & -4.9510161882 & -2.7204163287 \\ \mathrm{H} & 2.4457743644 & -3.1279959524 & -2.4677665803 \\ \mathrm{H} & 1.7861376199 & -1.8041992012 & -1.4963094597 \\ \mathrm{O} & 1.4774882361 & -0.7122999641 & 0.0000961819 \\ \mathrm{H} & -0.7712539600 & 1.3968164323 & 0.1956562968 \\ \mathrm{H} & -2.4482190906 & -0.0561974050 & 0.7724201582 \\ \mathrm{H} & -2.7277800558 & 0.3079429740 & -0.9212829820 \\ \mathrm{H} & -1.5466957780 & -2.3071262620 & 0.1960175004 \\ \mathrm{H} & -3.2274973629 & -2.0412252393 & -0.2294219259 \\ \mathrm{H} & -2.1946003621 & -3.3335377082 & -1.9758677624 \\ \mathrm{H} & -2.6108450960 & -1.7510247035 & -2.6378048822 \\ \mathrm{H} & 0.7702021180 & 0.9187851699 & -1.6865846069 \\ \mathrm{~N} & -0.1901739582 & 0.5814403719 & -1.6861853824 \\ \mathrm{H} & -0.7587419214 & 1.2665631672 & -2.1799065755 \\ \mathrm{Na} & 2.2329295877 & -1.1016381993 & 2.1651604851 \\ \mathrm{O} & 3.6538934487 & -1.4947043087 & 3.8989654296 \\ \mathrm{H} & 4.6177444073 & -1.5623842557 & 3.9199671886 \\ \mathrm{H} & 3.3690933485 & -1.5335651684 & 4.8226659342\end{array}$

\section{Arg $\cdot \mathrm{Na}^{+}\left(\mathrm{H}_{2} \mathrm{O}\right)$ Structure D1 *}

$\begin{array}{lrrr}\mathrm{C} & -0.3658536075 & 0.4082057451 & 0.0089428874 \\ \mathrm{C} & 0.4900251480 & -0.4237952493 & 0.9863641311 \\ \mathrm{O} & 1.6173939168 & -0.8635318629 & 0.5841623600 \\ \mathrm{C} & -1.8018513110 & -0.1487791386 & -0.1632362975 \\ \mathrm{C} & -2.0008043084 & -1.6461399341 & -0.4847858599 \\ \mathrm{C} & -1.5775815731 & -2.1433903074 & -1.8795639291 \\ \mathrm{~N} & -0.1254955722 & -2.0396881351 & -2.0597355097 \\ \mathrm{C} & 0.7576051066 & -3.0285084455 & -2.0233231037 \\ \mathrm{~N} & 0.4511902051 & -4.2719361414 & -2.4575973327 \\ \mathrm{~N} & 1.9870992337 & -2.7699466142 & -1.5477321529 \\ \mathrm{H} & 0.2325867689 & -1.0630247620 & -1.8843149131 \\ \mathrm{H} & -0.3216247368 & -4.4207707800 & -3.0880224817 \\ \mathrm{H} & 1.0637095750 & -5.0518018431 & -2.2747132979 \\ \mathrm{H} & 2.7586641372 & -3.3968196920 & -1.7172174160 \\ \mathrm{H} & 2.0794316083 & -2.0120670544 & -0.8621166850 \\ \mathrm{O} & 0.0189879548 & -0.5748909558 & 2.1449685020 \\ \mathrm{H} & -0.4777851311 & 1.3847797039 & 0.5019422242 \\ \mathrm{H} & -2.3214655394 & 0.0454538493 & 0.7794268876 \\ \mathrm{H} & -2.3092869208 & 0.4604748071 & -0.9243499874 \\ \mathrm{H} & -1.5002749907 & -2.2575597226 & 0.2765193224 \\ \mathrm{H} & -3.0734186106 & -1.8564081692 & -0.3895914942 \\ \mathrm{H} & -1.8925522854 & -3.1829194777 & -2.0026982747 \\ \mathrm{H} & -2.0661162364 & -1.5570055492 & -2.6658813391 \\ \mathrm{H} & -0.1115827457 & 1.2837258500 & -1.8458106659 \\ \mathrm{~N} & 0.3259206599 & 0.5529384830 & -1.2882864659 \\ \mathrm{H} & 1.2963441123 & 0.8195048578 & -1.1358810374 \\ \mathrm{Na} & 1.0947621669 & -1.1876955131 & 3.9227319754 \\ \mathrm{O} & 2.9463275705 & -1.5536674280 & 2.8066878793 \\ \mathrm{H} & 3.9089991833 & -1.5706136133 & 2.7840069088 \\ \mathrm{H} & 2.6018244687 & -1.3262055162 & 1.8969978742\end{array}$

$\begin{array}{ccrr}\text { Arg } \cdot \mathrm{Na}^{+}\left(\mathbf{H}_{\mathbf{2}} \mathbf{0}\right) \text { Structure } & \text { E1 } & \\ \text { C } & 0.0746224014 & -1.4721743445 & -0.0695612692 \\ \text { C } & 0.9224782711 & -1.0458612408 & 1.1527273214 \\ 0 & 0.8225897710 & 0.1467050601 & 1.5531840189 \\ \text { C } & -1.3420133904 & -0.8758396725 & -0.0400913820 \\ \text { C } & -2.3182925807 & -1.3676504791 & -1.1312920630\end{array}$




\begin{tabular}{|c|c|c|c|}
\hline C & -1.7971908143 & -1.3691264747 & -2.5772554377 \\
\hline $\mathrm{N}$ & $-\odot .8626172121$ & -2.4883237771 & -2.7799950764 \\
\hline C & -0.4895666751 & -2.9661530377 & -3.9499558536 \\
\hline $\mathrm{N}$ & $-\odot .8316360146$ & -2.3711570749 & -5.1119558441 \\
\hline $\mathrm{N}$ & 0.2453987756 & -4.1028596638 & -4.012238106 \\
\hline $\mathrm{H}$ & $-\odot .4444874599$ & -2.8925370667 & -1.884847224 \\
\hline $\mathrm{H}$ & -1.3366605782 & -1.4990036358 & -5.116901193 \\
\hline $\mathrm{H}$ & $-\odot .7429670372$ & -2.8603054162 & -5.989921843 \\
\hline $\mathrm{H}$ & 0.8065324225 & -4.3040257137 & $-4.826486927 i$ \\
\hline $\mathrm{H}$ & $\odot .5045465835$ & -4.5685915261 & -3.154628408 \\
\hline 0 & 1.7039902732 & -1.9177876156 & 1.643926401 \\
\hline $\mathrm{H}$ & 0.6075603821 & -1.0516950668 & -0.936326148 \\
\hline $\mathrm{H}$ & -1.8003713010 & -1.0826546771 & 0.935162666 \\
\hline $\mathrm{H}$ & -1.2340283444 & 0.2110228719 & $-\odot .090821592$ \\
\hline $\mathrm{H}$ & -2.67974 & -2.3 & $-0.9 \odot 4177390$ \\
\hline $\mathrm{H}$ & -3.2029717637 & -0.7223459295 & -1.105619333 \\
\hline $\mathrm{H}$ & -2.6455492070 & -1.4883755688 & -3.262137990 \\
\hline $\mathrm{H}$ & -1.2991305000 & -0.4164610997 & -2.807563940 \\
\hline $\mathrm{H}$ & $-\odot .6155293456$ & -3.3599478716 & 0.382344955 \\
\hline $\mathrm{N}$ & ๑. 0595801248 & -2.9431163245 & $-\odot .259621089 \varsigma$ \\
\hline H & 0.9662816173 & -3.2829986709 & 0.070068414 \\
\hline $\mathrm{Na}$ & 2.4237094212 & -0.3203211002 & 3. 081413886 \\
\hline 0 & 3.9019826574 & $\odot .2815987700$ & 4.716490938 \\
\hline $\mathrm{H}$ & 3. 9911722588 & 1.1484769859 & 5.134688260 \\
\hline & 4.5665986822 & -0.2821710488 & \\
\hline
\end{tabular}

\begin{tabular}{|c|c|c|c|}
\hline \multicolumn{2}{|c|}{ Arg $\cdot \mathrm{Na}^{+}\left(\mathrm{H}_{2} \mathrm{O}\right)$ Structure } & \multicolumn{2}{|l|}{ E1* } \\
\hline $\mathrm{C}$ & 0.1585878942 & -0.4768230385 & 0.1848321733 \\
\hline C & $\odot .8789793981$ & -0.4123703423 & 1.5560669362 \\
\hline 0 & 2.0154552522 & -0.9607278181 & 1.6259921788 \\
\hline C & -1.0425562480 & -1.4408816866 & $\odot .2902750948$ \\
\hline C & -2.0100051635 & -1.4438771716 & -0.9161321550 \\
\hline C & -1.7532715015 & -2.5149116432 & -1.9840735943 \\
\hline $\mathrm{N}$ & -0.4271165864 & -2.3519871172 & -2.5909431873 \\
\hline C & -0.0251005325 & -2.9642936180 & -3.6862728125 \\
\hline $\mathrm{N}$ & -0.7840418636 & -3.8857695335 & -4.3165742403 \\
\hline $\mathrm{N}$ & 1.1789612910 & -2.6594821813 & -4.2305884380 \\
\hline $\mathrm{H}$ & $\odot .2697068900$ & -1.7584752574 & -2.0257295344 \\
\hline $\mathrm{H}$ & -1.6733461374 & -4.1694546072 & -3.9367869342 \\
\hline $\mathrm{H}$ & -0.5698893523 & -4.1767206317 & -5.2584138420 \\
\hline $\mathrm{H}$ & 1.6381636102 & -3.3129925128 & -4.8473700982 \\
\hline $\mathrm{H}$ & 1.7687918349 & -1.9839397782 & -3.7677345410 \\
\hline 0 & 0.2536637354 & 0.1628706797 & 2.4923662331 \\
\hline $\mathrm{H}$ & $-\odot .2287220274$ & 0.5247424689 & $-\odot .0299056087$ \\
\hline $\mathrm{H}$ & -0.6759218363 & -2.4583656856 & 0.4873306587 \\
\hline $\mathrm{H}$ & -1.5975986808 & -1.1372156308 & 1.1822264953 \\
\hline $\mathrm{H}$ & -3.0282715141 & -1.6219939656 & -0.5535648962 \\
\hline $\mathrm{H}$ & -2.0256764263 & -0.4556348358 & -1.3928432137 \\
\hline $\mathrm{H}$ & -1.8314734713 & -3.5122254465 & -1.5275682470 \\
\hline $\mathrm{H}$ & -2.5217351137 & -2.4338108942 & -2.7631378906 \\
\hline $\mathrm{H}$ & 1.8437715932 & -1.3976509971 & -0.4248426824 \\
\hline $\mathrm{N}$ & 1.1050176469 & $-\odot .8637624501$ & $-\odot .8965559860$ \\
\hline $\mathrm{H}$ & 1.5736834694 & -0.0236788153 & -1.2331877660 \\
\hline $\mathrm{Na}$ & 1.1413834792 & ๑.3886589703 & 4.4625327686 \\
\hline 0 & 3. 0077426378 & -0.6585279442 & 4.0532530787 \\
\hline $\mathrm{H}$ & 3.8827985342 & -0.9978888991 & 4.2667942012 \\
\hline $\mathrm{H}$ & 2.7949773641 & -0.8704274558 & 3.0969273635 \\
\hline
\end{tabular}




\section{Arg $\cdot \mathrm{Na}^{+}\left(\mathrm{H}_{2} \mathrm{O}\right)$ Structure F1}

$\begin{array}{lrr}\mathrm{C} & -1.3716337470 & -2.2422443151 \\ \mathrm{C} & -2.0974326042 & -0.8901004243 \\ \mathrm{C} & -3.6278396098 & -0.9582173767 \\ \mathrm{C} & -4.4269255520 & -1.3309901590 \\ \mathrm{~N} & -4.3042115139 & -0.3469550039 \\ \mathrm{C} & -3.5646019806 & -0.4743078391 \\ \mathrm{~N} & -2.6891599473 & -1.4615445986 \\ \mathrm{~N} & -3.7371384638 & 0.4416008819 \\ \mathrm{H} & -4.7901354494 & 0.5313271279 \\ \mathrm{H} & -2.2890842769 & -2.0342720592 \\ \mathrm{H} & -2.2655913842 & -1.5991045022 \\ \mathrm{H} & -3.1105086196 & 0.4730887145 \\ \mathrm{H} & -4.5747390496 & 1.0017897477 \\ \mathrm{H} & -1.7183772034 & -2.8885212385 \\ \mathrm{H} & -1.8125677776 & -0.2436747394 \\ \mathrm{H} & -1.7004540706 & -0.4100360799 \\ \mathrm{H} & -3.9932218038 & 0.0139863883 \\ \mathrm{H} & -3.8992990952 & -1.6814170620 \\ \mathrm{H} & -5.4916592629 & -1.3922844316 \\ \mathrm{H} & -4.1371596870 & -2.3034302067 \\ \mathrm{C} & 0.1509717977 & -2.0604541906 \\ \mathrm{O} & 0.5154553422 & -1.4638303983 \\ \mathrm{O} & 0.9398297609 & -2.5421800087 \\ \mathrm{~N} & -1.5941479153 & -2.9212882491 \\ \mathrm{H} & -0.6492946359 & -3.1603007107 \\ \mathrm{H} & -2.0772681717 & -3.8069461709 \\ \mathrm{Na} & 2.6535220453 & -1.9017572136 \\ \mathrm{O} & 4.8392873173 & -1.7469895340 \\ \mathrm{H} & 5.1560280325 & -1.3386340450 \\ \mathrm{H} & 5.6239695714 & -2.1066529925\end{array}$

\section{Arg $\cdot \mathrm{Na}^{+}\left(\mathrm{H}_{2} \mathbf{0}\right)$ structure $\mathbf{G 1}$}

\begin{tabular}{|c|c|}
\hline & - ๑. . 0403657684 \\
\hline$C$ & 1.0288510072 \\
\hline 0 & 1.8505833534 \\
\hline$C$ & $-\odot .785418290 \odot$ \\
\hline C & -1.9504470207 \\
\hline C & -1.8244675133 \\
\hline $\mathrm{N}$ & -0.6120614214 \\
\hline C & $-\odot .2817858310$ \\
\hline $\mathrm{N}$ & -0.9688221758 \\
\hline $\mathrm{N}$ & 0.7828244201 \\
\hline $\mathrm{H}$ & ๑.0382656860 \\
\hline $\mathrm{H}$ & -1.7691198390 \\
\hline $\mathrm{H}$ & -0.8439804221 \\
\hline $\mathrm{H}$ & 1.2679266077 \\
\hline $\mathrm{H}$ & 1.3031198309 \\
\hline 0 & ๑.9787710843 \\
\hline $\mathrm{H}$ & -0.7799436560 \\
\hline $\mathrm{H}$ & -1.1983837611 \\
\hline $\mathrm{H}$ & -0.0536005413 \\
\hline $\mathrm{H}$ & -2.0350626450 \\
\hline $\mathrm{H}$ & -2.9013772251 \\
\hline $\mathrm{H}$ & -2.7064433855 \\
\hline H & -1.774003560 \\
\hline
\end{tabular}

$-0.5341092755$

$-1.6813813040$

$-1.7712736497$

$-0.1995225208$

$-1.1614952846$

$-1.8067401755$

$-2.6203067738$

$-3.5692874552$

$-3.7986380870$

$-4.3542235800$

$-2.4876742547$

$-3.2340589248$

$-4.6596074239$

$-4.8467523432$

$-4.1505537294$

$-2.4595097405$

$-0.8917515463$

0.8101066045

$-0.1395405489$

$-1.9666931538$

$-0.6201683084$

$-2.4300798171$

$-1.0301167519$
1.4396646576

1.3140574333

1.1825660862

2. 4414329902

3. 5313631405

4.6438704140

4.7827414585

5. 6286955751

3. 4073167928

3. 9532431733

5. 6901282762

6.4180441098

5.6818188544

0.6222158391

2. 1556889413

0.4152481460

0.8262674632

0.4018993637

2. 1940683437

2. 8411508043

1. 2085019403

0.1605534265

2. 0827693236

2. 7472632780

3. 0759118003

2. 6101090947

0.7381616302

0.1007740592

$-0.7162815761$

0.5361437354
0.6526171223

0.5533260169

1.4947107050

$-0.6644707158$

$-1.0427178734$

$-2.4316089670$

$-2.4435872871$

$-3.2925417631$

$-4.4346658839$

$-3.0042662744$

$-1.6012690647$

$-4.6721261951$

$-4.9452004063$

$-3.7389461761$

$-2.1563411371$

$-0.4445780441$

1.3829636820

$-0.5587314598$

$-1.4800818200$

$-0.3031754088$

$-1.0168577460$

$-2.6295657791$

$-3.2081553333$ 


$\begin{array}{lrrr}\mathrm{H} & 1.2124310649 & 1.1018363270 & 0.5900095559 \\ \mathrm{~N} & 0.5988179460 & 0.6583378037 & 1.2753166452 \\ \mathrm{H} & -0.1180236364 & 1.3522190639 & 1.4829852774 \\ \mathrm{Na} & 2.0949022328 & -0.2461770454 & 2.9939050898 \\ \mathrm{O} & 3.2127349851 & 0.4807660757 & 4.8467992099 \\ \mathrm{H} & 3.1054194936 & 1.2926428268 & 5.3601721984 \\ \mathrm{H} & 3.9204745040 & -0.0132228894 & 5.2829049059\end{array}$

\section{Arg $\cdot \mathrm{Na}^{+}\left(\mathrm{H}_{2} \mathrm{O}\right)$ Structure $\mathrm{G1}^{\text {* }}$}

$\begin{array}{lrr}\mathrm{C} & -0.5111444302 & -0.2255303602 \\ \mathrm{C} & 0.7652619172 & -1.1334968535 \\ \mathrm{O} & 1.0825491393 & -1.8495672822 \\ \mathrm{C} & -1.1910844375 & -0.1693973884 \\ \mathrm{C} & -2.0295252340 & -1.4267088525 \\ \mathrm{C} & -1.6432160801 & -2.0770466620 \\ \mathrm{~N} & -0.2586429628 & -2.5356644045 \\ \mathrm{C} & 0.3463961810 & -3.4433784965 \\ \mathrm{~N} & -0.1936920119 & -3.9331370112 \\ \mathrm{~N} & 1.5491094112 & -3.9112371426 \\ \mathrm{H} & 0.2777142329 & -2.1837121297 \\ \mathrm{H} & -1.0733084805 & -3.5826744898 \\ \mathrm{H} & 0.1743452256 & -4.7674348187 \\ \mathrm{H} & 2.1837682678 & -4.3380716412 \\ \mathrm{H} & 1.9419169082 & -3.5375100783 \\ \mathrm{O} & 1.3899823243 & -1.0842156108 \\ \mathrm{H} & -1.2243927203 & -0.6512998219 \\ \mathrm{H} & -1.8544150572 & 0.7032016676 \\ \mathrm{H} & -0.4254574324 & 0.0282561533 \\ \mathrm{H} & -1.9438150976 & -2.1899803347 \\ \mathrm{H} & -3.0902347327 & -1.1631957322 \\ \mathrm{H} & -2.3103753441 & -2.9244040945 \\ \mathrm{H} & -1.7489590336 & -1.3530793340 \\ \mathrm{H} & 0.4247990462 & 1.6068992365 \\ \mathrm{~N} & -0.1556365127 & 1.1219175457 \\ \mathrm{H} & -1.0019640939 & 1.6841595053 \\ \mathrm{Na} & 1.2729106438 & 0.6992619192 \\ \mathrm{O} & 3.3455565421 & -0.0953727695 \\ \mathrm{H} & 4.2115603158 & -0.2304632938 \\ \mathrm{H} & 3.1674939383 & -0.8308412516\end{array}$

0.7887888258

0.8810450788

$-0.1097074754$

$-\odot .5977437583$

$-0.9723440241$

$-2.3098492934$

$-2.2107674650$

$-2.9485900066$

$-4.0859134071$

$-2.5430951051$

$-1.3615589962$

$-4.4306465056$

$-4.5165135525$

$-3.2006683317$

$-1.6851254436$

1.9713076150

1.5076081792

$-0.6054186843$

$-1.3588806548$

$-\odot .1894894409$

$-1.0277010014$

$-2.5141019591$

$-3.1300185268$

$\odot .6329297787$

1.3191186888

1.3991469218

3. 2654228411

3.5384380147

3.9381454718

2. 9227176825

\section{Arg $\cdot \mathrm{Na}^{+}\left(\mathrm{H}_{2} \mathrm{O}\right)$ Structure $\mathrm{H} 1$}

$\begin{array}{lrrr}\mathrm{C} & -1.0951637164 & -0.6816636704 & 0.2524654110 \\ \mathrm{C} & 0.3623444954 & -0.7696940101 & 0.7530717842 \\ \mathrm{O} & 0.7465444171 & -0.0444220071 & 1.6945003844 \\ \mathrm{C} & -1.1544774225 & 0.1315095900 & -1.0852912050 \\ \mathrm{C} & -1.7643443237 & -0.6229406128 & -2.2836425487 \\ \mathrm{C} & -0.7669282851 & -1.4967075565 & -3.0646117784 \\ \mathrm{~N} & -0.0240680927 & -2.4219825854 & -2.1989735833 \\ \mathrm{C} & -0.2657595666 & -3.7084491787 & -1.9850134373 \\ \mathrm{~N} & -0.9649443808 & -4.4739596274 & -2.8458611462 \\ \mathrm{~N} & 0.2140959225 & -4.2617546863 & -0.8527308314 \\ \mathrm{H} & 0.5390538087 & -1.9875991786 & -1.4384269690 \\ \mathrm{H} & -1.2435195650 & -4.1249333151 & -3.7489309408 \\ \mathrm{H} & -1.2706964721 & -5.3993585730 & -2.5875772794 \\ \mathrm{H} & 0.3438189966 & -5.2585510483 & -0.7700353583 \\ \mathrm{H} & 0.6732155991 & -3.6398751047 & -0.1889240909 \\ \mathrm{O} & 1.1601492525 & -1.5953674387 & 0.1375658529\end{array}$




$\begin{array}{lr}\mathrm{H} & -1.4191752249 \\ \mathrm{H} & -1.7775333010 \\ \mathrm{H} & -0.1632206609 \\ \mathrm{H} & -2.6121163440 \\ \mathrm{H} & -2.1696281374 \\ \mathrm{H} & -1.2817052144 \\ \mathrm{H} & -0.0228981882 \\ \mathrm{H} & -2.1308659823 \\ \mathrm{~N} & -2.0414937124 \\ \mathrm{H} & -1.6968175509 \\ \mathrm{Na} & 2.8600619510 \\ \mathrm{O} & 4.9602607401 \\ \mathrm{H} & 5.1524984884 \\ \mathrm{H} & 5.8204527487\end{array}$

$-1.7100565991$

1. 0062307932

0.5160451913

$-1.2294768085$

0.0999790689

$-2.0500409490$

$-0.8644098265$

$-0.7155907361$

$-0.1180733485$

0.7776619787

$-0.7369243142$

$-0.2616126053$

$\odot .3516513067$

$-0.5649226296$
0.0537544495

$-\odot .8800713096$

$-1.3607017792$

$-1.9419863423$

$-3.0016582846$

$-3.8550044356$

$-3.5602622275$

2. 0232460376

1. 2052056500

1.5459983600

1.4638935890

2.1913840602

2. 9143457687

1.8711797536

\section{Arg $\cdot \mathrm{Na}^{+}\left(\mathrm{H}_{2} \mathrm{O}\right)$ Structure I1}

\begin{tabular}{|c|c|c|c|}
\hline C & -1.3384995875 & -1.2595278081 & $-\odot .5591438566$ \\
\hline C & -2.1499064637 & -1.3691609066 & 0.7543929904 \\
\hline C & -1.5210990952 & $-\odot .6891974911$ & 1.9854417265 \\
\hline & -1.3213603613 & ๑. 8283380954 & 1.8312981626 \\
\hline $\mathrm{N}$ & -0.5285273820 & 1.4118051204 & 2.9234481518 \\
\hline C & 0.8107638451 & 1.5262534161 & 2.9394268740 \\
\hline $\mathrm{N}$ & 1.5725026894 & 1.0645295163 & 1.9603907569 \\
\hline $\mathrm{N}$ & 1.3750916632 & 2.1298139443 & 4.0128652008 \\
\hline H & -1.0075091910 & 1.5950149467 & 3.7940170081 \\
\hline $\mathrm{H}$ & 1.2358415522 & ๑.4455185853 & 1.1751319351 \\
\hline $\mathrm{H}$ & 2.5569353745 & 1.2919238585 & 1.9580636210 \\
\hline $\mathrm{H}$ & 2.3788638922 & 2.1999786937 & 4.0840135046 \\
\hline $\mathrm{H}$ & 0.8381969119 & 2.7637351694 & 4.5791435754 \\
\hline $\mathrm{H}$ & -1.4381523029 & - ๑. 2467674856 & $-\odot .9598450910$ \\
\hline $\mathrm{H}$ & -3.1535854576 & -0.9612496244 & 0.5777399829 \\
\hline $\mathrm{H}$ & 26547060 & -2.4360528492 & 0.9856418673 \\
\hline $\mathrm{H}$ & 52356311 & -0.8777167879 & 2.8518242095 \\
\hline $\mathrm{H}$ & -0.5563678812 & -1.1613876015 & 2.2045574698 \\
\hline $\mathrm{H}$ & -0.8066035124 & 1.0595971012 & 0.8990495270 \\
\hline $\mathrm{H}$ & -2.2815304865 & 1.3524918681 & 1.8233265194 \\
\hline C & 0.1722041054 & -1.4994846511 & $-\odot .2766514135$ \\
\hline 0 & 0.821977 & -0.4642190288 & ๑.0206855546 \\
\hline 0 & 0.612 & -2.6752565816 & $-\odot .3251582792$ \\
\hline $\mathrm{N}$ & -1.8679127625 & -2.2339434458 & -1.5477773682 \\
\hline $\mathrm{H}$ & -1.5825521847 & -1.9405030959 & -2.4810756973 \\
\hline $\mathrm{H}$ & -2.8870791303 & -2.1920899513 & -1.5543520345 \\
\hline $\mathrm{Na}$ & -0.5516219833 & -4.2691174263 & -1.1928023864 \\
\hline 0 & -0.1306136591 & -6.4199756277 & -1.8212585487 \\
\hline $\mathrm{H}$ & 0.7317424517 & -6.7927685481 & -1.7462542722 \\
\hline $\mathrm{H}$ & $-\odot .7165244656$ & -7.1069134998 & -2.2174158157 \\
\hline
\end{tabular}

\section{Arg $\cdot \mathrm{Na}^{+}\left(\mathrm{H}_{2} \mathrm{O}\right)$ Structure $\mathrm{J1}$}

$\begin{array}{lr}\mathrm{C} & -0.5944922495 \\ \mathrm{C} & 0.4465026034 \\ \mathrm{O} & 1.1911564962 \\ \mathrm{C} & -1.9827448548 \\ \mathrm{C} & -2.1041515489 \\ \mathrm{C} & -1.7262749040 \\ \mathrm{~N} & -0.2683044998 \\ \mathrm{C} & 0.3739523006 \\ \mathrm{~N} & -0.2466306410\end{array}$

0.4409557566

$-0.1776956639$

$-1.1487425775$

$-0.2242673592$

$-1.7635466320$

$-2.5205103289$

$-2.6203797722$

$-2.8921060355$

$-3.4578686703$ $-\odot .3654945217$
0.5849127908
0.1661736538
-0.1841034572
-0.1839522324
-1.4677688707
-1.5996096708
-2.7210173096
-3.7773936240 


$\begin{array}{lrrr}\mathrm{N} & 1.6888090971 & -2.6004996342 & -2.7962853271 \\ \mathrm{H} & 0.2972820098 & -2.0927844780 & -0.8992205881 \\ \mathrm{H} & -1.1727044095 & -3.8458005976 & -3.6790153142 \\ \mathrm{H} & 0.2454288452 & -3.6462305090 & -4.6371897988 \\ \mathrm{H} & 2.2430329854 & -2.8320224821 & -3.6059021896 \\ \mathrm{H} & 2.0710559383 & -1.9599985868 & -2.1106618937 \\ \mathrm{O} & 0.5098225249 & 0.2710011704 & 1.7531364772 \\ \mathrm{H} & -0.7079748566 & 1.4785115430 & -0.0231247866 \\ \mathrm{H} & -2.3835563022 & 0.1271911320 & 0.7729864062 \\ \mathrm{H} & -2.6433176431 & 0.1875563660 & -0.9596029134 \\ \mathrm{H} & -1.5400921472 & -2.1920270191 & 0.6551795127 \\ \mathrm{H} & -3.1549929184 & -1.9972170105 & 0.0195636946 \\ \mathrm{H} & -2.1555138507 & -3.5301067680 & -1.4206417478 \\ \mathrm{H} & -2.1426740256 & -2.0097477077 & -2.3468459867 \\ \mathrm{H} & 0.7321806856 & 0.8431191334 & -1.8842014491 \\ \mathrm{~N} & -0.1556272704 & 0.3584119076 & -1.7698923148 \\ \mathrm{H} & -0.8227954037 & 0.8567355973 & -2.3565384328 \\ \mathrm{Na} & 2.1738151430 & -1.1350059478 & 2.2590466249 \\ \mathrm{O} & 3.5389443354 & -1.4835423322 & 4.0471380161 \\ \mathrm{H} & 4.2710722039 & -2.0973160979 & 4.1829420051 \\ \mathrm{H} & 3.4708684703 & -0.9692502726 & 4.8707379166\end{array}$

\section{Arginine methyl ester $\bullet \mathrm{Li}^{+}\left(\mathrm{H}_{2} \mathrm{O}\right)\left(\operatorname{ArgOMe} \cdot \mathrm{Li}^{+}\left(\mathrm{H}_{2} \mathrm{O}\right)\right)$}

\section{ArgoMe $\bullet \mathrm{Li}^{+}\left(\mathrm{H}_{2} \mathrm{O}\right)$ Structure A1}

\begin{tabular}{|c|c|c|c|}
\hline C1 & 0.8289442264 & 0.6648070674 & -1.7545566580 \\
\hline C2 & 1. 0825866065 & 1.3331599780 & -3.1303953982 \\
\hline C3 & $\odot .8579955201$ & 2.8569378242 & -3.2048007070 \\
\hline C4 & -0.6075893990 & 3.2806190091 & -3.4383937592 \\
\hline N5 & $-\odot .8418560683$ & 4.7022775883 & -3.2336016925 \\
\hline C6 & -1.0380150611 & 5.2455916145 & -1.9936496253 \\
\hline N7 & -1.1858040195 & 6.6164042100 & -1.9972755536 \\
\hline N8 & -1.0531224710 & 4.5033595241 & -0.9177031966 \\
\hline H9 & -0.5458380857 & 5.3331366725 & -3.9651020881 \\
\hline H10 & -1.5869811024 & 7.0580137575 & -2.8132563754 \\
\hline H11 & -1.4177511215 & 7.0653112681 & 7401099 \\
\hline H12 & -1.3181075328 & 5.0660810850 & -0.1111733595 \\
\hline H13 & 1.1256080300 & -0.3882096419 & -1.8450355239 \\
\hline H14 & 2.1171567990 & 1.1045568784 & -3.4036126245 \\
\hline H15 & 0.4564805336 & ๑.8172345846 & -3.8711142645 \\
\hline H16 & 1.2251578547 & 3. 3442850904 & -2.2943003238 \\
\hline H17 & 1.4661064842 & 3.2558665535 & -4.0249655489 \\
\hline H18 & -0.9093332413 & 3.0379552066 & -4.4629555256 \\
\hline H19 & -1.2852751853 & 2.7496011157 & -2.7685424031 \\
\hline N20 & -0.5713020380 & ๑.8215161680 & -1.3083550192 \\
\hline H21 & -1.2021892797 & 0.5730167243 & -2.0672651581 \\
\hline H22 & -0.7721241686 & 0.164 & 0681864 \\
\hline C23 & 1.7 & 1.29 & 4710203 \\
\hline 024 & 2.9721312517 & $\odot .9004385307$ & -0.7588078899 \\
\hline 025 & 1.2928930085 & 2.1018288750 & 0.1460155164 \\
\hline Li26 & -0.5899737335 & 2.6978225092 & -0.2262030212 \\
\hline 027 & -1.3870686847 & 2.4474012289 & 1.5621732240 \\
\hline H28 & $-\odot .8239521036$ & 2.3164431787 & $2.33659 \odot 2675$ \\
\hline H29 & -2.2843072856 & 2.5898780742 & 1.8894002859 \\
\hline C30 & 3.9187557309 & 1.4663732768 & 0.1885623157 \\
\hline H31 & 4.8787974824 & $1.028090700 \odot$ & -0.0764806233 \\
\hline
\end{tabular}




$\begin{array}{llll}\text { H32 } & 3.9410771200 & 2.5526229874 & 0.0873243441 \\ \text { H33 } & 3.6313297307 & 1.1906415001 & 1.2046741184\end{array}$

\begin{tabular}{crrr}
\multicolumn{4}{c}{ ArgOMe Li $^{+}\left(\mathbf{H}_{\mathbf{2}} \mathbf{0}\right)$ Structure C1 } \\
$\mathrm{C}$ & 2.4513109231 & -0.2374725298 & -2.4748022543 \\
$\mathrm{C}$ & 3.0738368736 & 1.1771507020 & -2.5977820011 \\
$\mathrm{C}$ & 2.2311697062 & 2.3374822058 & -3.1725660210 \\
$\mathrm{C}$ & 2.0637742316 & 2.4605635869 & -4.6984493934 \\
$\mathrm{~N}$ & 0.9794773955 & 1.6463943657 & -5.2573140719 \\
$\mathrm{C}$ & 0.6093367720 & 1.9220472655 & -6.4768178299 \\
$\mathrm{H}$ & 2.0622188777 & 3.2794679283 & -7.0230055470 \\
$\mathrm{H}$ & 0.9565707283 & 2.9020158293 & -8.2591926719 \\
$\mathrm{H}$ & -0.9044999091 & 1.6499206044 & -7.8337766326 \\
$\mathrm{H}$ & -1.0278446831 & 0.7221423215 & -6.4231196353 \\
$\mathrm{H}$ & 3.1232037381 & -0.8280612209 & -1.8403321684 \\
$\mathrm{H}$ & 3.3807645059 & 1.4593401390 & -1.5854270112 \\
$\mathrm{H}$ & 4.0043482830 & 1.0734327724 & -3.1726619285 \\
$\mathrm{H}$ & 1.2389986636 & 2.3737872961 & -2.7057350442 \\
$\mathrm{H}$ & 2.7398650409 & 3.2525553915 & -2.8483321839 \\
$\mathrm{H}$ & 1.8674998583 & 3.5246438192 & -4.8977668024 \\
$\mathrm{H}$ & 3.0295087481 & 2.2233584221 & -5.1805987625 \\
$\mathrm{Li}$ & 0.4063601166 & -0.0296888959 & -4.3334971702 \\
$\mathrm{~N}$ & 2.2316741434 & -0.8687985725 & -3.7920748425 \\
$\mathrm{H}$ & 3.0124911127 & -0.6625346722 & -4.4108503096 \\
$\mathrm{H}$ & 2.2017453398 & -1.8825600667 & -3.6962770524 \\
$\mathrm{C}$ & 1.0991457531 & -0.1544740928 & -1.7681054413 \\
$\mathrm{O}$ & 1.2122358560 & -0.0790577371 & -0.4546152778 \\
$\mathrm{O}$ & 0.0287781553 & -0.1251577196 & -2.3725952869 \\
$\mathrm{~N}$ & -0.4157996424 & 1.2068318762 & -7.0674650012 \\
$\mathrm{~N}$ & 1.1672241226 & 2.8899090802 & -7.2722580916 \\
$\mathrm{O}$ & -1.1749519142 & -1.0216834986 & -5.0410574642 \\
$\mathrm{H}$ & -1.8440142398 & -1.2185909555 & -4.3699950412 \\
$\mathrm{H}$ & -1.1933769899 & -1.7534864841 & -5.6728284021 \\
$\mathrm{C}$ & -0.0071441338 & 0.0641737700 & 0.3237978629 \\
$\mathrm{H}$ & 0.3256915008 & 0.1326441528 & 1.3573961339 \\
$\mathrm{H}$ & -0.5369436192 & 0.9687075301 & 0.0207185587 \\
$\mathrm{H}$ & -0.6426626803 & -0.8105683641 & 0.1757108591 \\
& & &
\end{tabular}

